\title{
Covering of High-Dimensional Cubes and Quantization
}

\author{
Anatoly Zhigljavsky ${ }^{1}$. Jack Noonan ${ }^{1}$
}

Received: 24 February 2020 / Accepted: 14 May 2020 / Published online: 13 August 2020

(C) The Author(s) 2020

\begin{abstract}
As the main problem, we consider covering of a $d$-dimensional cube by $n$ balls with reasonably large $d$ (10 or more) and reasonably small $n$, like $n=100$ or $n=1000$. We do not require the full coverage but only $90 \%$ or $95 \%$ coverage. We establish that efficient covering schemes have several important properties which are not seen in small dimensions and in asymptotical considerations, for very large $n$. One of these properties can be termed 'do not try to cover the vertices' as the vertices of the cube and their close neighbourhoods are very hard to cover and for large $d$ there are far too many of them. We clearly demonstrate that, contrary to a common belief, placing balls at points which form a low-discrepancy sequence in the cube, results in a very inefficient covering scheme. For a family of random coverings, we are able to provide very accurate approximations to the coverage probability. We then extend our results to the problems of coverage of a cube by smaller cubes and quantization, the latter being also referred to as facility location. Along with theoretical considerations and derivation of approximations, we provide results of a large-scale numerical investigation.
\end{abstract}

Keywords Covering · Quantization · Facility location · Space-filling · Computer experiments · High dimension

Mathematics Subject Classification (2010) 90C26 · 65K99 • 65B99

Anatoly Zhigljavsky

ZhigljavskyAA@cardiff.ac.uk

Jack Noonan

Noonanj1@ cardiff.ac.uk

1 School of Mathematics, Cardiff University, Cardiff, CF244AG, UK 


\section{Introduction}

In this paper, we develop and study efficient schemes for covering and quantization in high-dimensional cubes. In particular, we will demonstrate that the proposed schemes are much superior to the so-called low-discrepancy sequences. The paper starts with introducing the main notation, then we formulate the main problem of covering a $d$-dimensional cube by $n$ Euclidean balls. This is followed by a discussion on the main principles we have adopted for construction of our algorithms. Then we briefly formulate problems of covering a cube by smaller cubes (which are balls in the $L_{\infty^{-}}$ norm) and the problem of quantization. Both problems have many similarities with the main problem of covering a cube by $n$ balls. At the end of this section, we describe the structure of the remaining sections of the paper and summarize our main findings.

\subsection{Main Notation}

- $\quad \mathbb{R}^{d}: d$-dimensional space; vectors in $\mathbb{R}^{d}$ are row-vectors;

- $\|\cdot\|$ and $\|\cdot\|_{\infty}$ : Euclidean and $L_{\infty}$-norms in $\mathbb{R}^{d}$;

- $\mathcal{B}_{d}(Z, r)=\left\{Y \in \mathbb{R}^{d}:\|Y-Z\| \leq r\right\}: d$-dimensional ball of radius $r$ centred at $Z \in \mathbb{R}^{d}$

- $\mathcal{B}_{d}(r)=\mathcal{B}_{d}(0, r)=\left\{Y \in \mathbb{R}^{d}:\|Y\| \leq r\right\}$

- $\mathcal{S}_{d}(Z, r)=\left\{Y \in \mathbb{R}^{d}:\|Y-Z\|=r\right\}: d$-dimensional sphere of radius $r$ centred at $Z \in \mathbb{R}^{d}$;

- $\mathcal{C}_{d}(Z, \delta)=\left\{Y \in \mathbb{R}^{d}:\|Y-Z\|_{\infty} \leq \delta\right\}: d$-dimensional cube of side length $2 \delta$ centred at $Z$ (it is also the $d$-dimensional ball in the $L_{\infty}$-norm with radius $\delta$ and centre $Z$ );

$-\mathcal{C}_{d}(\delta)=[-\delta, \delta]^{d}=\mathcal{C}_{d}(0, \delta)$

$-\mathcal{C}_{d}=[-1,1]^{d}=\mathcal{C}_{d}(1)$.

\subsection{Main Problem of Interest}

The main problem discussed in the paper is the following problem of covering a cube by $n$ balls. Let $\mathcal{C}_{d}=[-1,1]^{d}$ be a $d$-dimensional cube, $Z_{1}, \ldots, Z_{n}$ be some points in $\mathbb{R}^{d}$ and $\mathcal{B}_{d}\left(Z_{j}, r\right)$ be the corresponding balls of radius $r$ centred at $Z_{j}(j=1, \ldots, n)$. The dimension $d$, the number of balls $n$ and their radius $r$ could be arbitrary.

We are interested in the problem of choosing the locations of the centres of the balls $Z_{1}, \ldots, Z_{n}$ so that the union of the balls $\cup_{j} \mathcal{B}_{d}\left(Z_{j}, r\right)$ covers the largest possible proportion of the cube $\mathcal{C}_{d}$. That is, we are interested in choosing a design (a collection of points) $\mathbb{Z}_{n}=\left\{Z_{1}, \ldots, Z_{n}\right\}$ so that

$$
C_{d}\left(\mathbb{Z}_{n}, r\right):=\operatorname{vol}\left(\mathcal{C}_{d} \cap \mathcal{B}_{d}\left(\mathbb{Z}_{n}, r\right)\right) / 2^{d}
$$

is as large as possible (given $n, r$ and the freedom we are able to use in choosing $\left.Z_{1}, \ldots, Z_{n}\right)$. Here

$$
\mathcal{B}_{d}\left(\mathbb{Z}_{n}, r\right)=\bigcup_{j=1}^{n} \mathcal{B}_{d}\left(Z_{j}, r\right)
$$


and $C_{d}\left(\mathbb{Z}_{n}, r\right)$ is the proportion of the cube $\mathcal{C}_{d}$ covered by the balls $\mathcal{B}_{d}\left(Z_{j}, r\right)(j=$ $1, \ldots, n)$. If points $Z_{j} \in \mathbb{Z}_{n}$ are random then by $C_{d}\left(\mathbb{Z}_{n}, r\right)$ we will mean $\mathbb{E}_{\mathbb{Z}_{n}} C_{d}\left(\mathbb{Z}_{n}, r\right)$ but we are not going to stress this in notation unless it is important.

For a design $\mathbb{Z}_{n}$, its covering radius is defined by $\mathrm{CR}\left(\mathbb{Z}_{n}\right)=$ $\max _{X \in \mathcal{C}_{d}} \min _{Z_{j} \in \mathbb{Z}_{n}}\left\|X-Z_{j}\right\|$. In computer experiments, covering radius is called minimax-distance criterion (see [5] and [13]); in the theory of low-discrepancy sequences, covering radius is called dispersion (see [8, Ch. 6]).

The problem of optimal covering of a cube by $n$ balls has very high importance for the theory of global optimization and many branches of numerical mathematics. In particular, the $n$-point designs $\mathbb{Z}_{n}$ with smallest CR provide the following: (a) the $n$ point min-max optimal quadratures (see [14, Ch.3,Th.1.1]), (b) min-max $n$-point global optimization methods in the set of all adaptive $n$-point optimization strategies (see [14, Ch.4,Th.2.1]), and (c) worst-case $n$-point multi-objective global optimization methods in the set of all adaptive $n$-point algorithms (see [20]). In all three cases, the class of (objective) functions is the class of Liptshitz functions, where the Liptshitz constant may be unknown. The results (a) and (b) are the celebrated results of A.G.Sukharev obtained in the late nineteen-sixties and (c) is a recent result of A. Žilinskas.

If $d$ is not small (say, $d>5$ ) then computation of the covering radius $\mathrm{CR}\left(\mathbb{Z}_{n}\right)$ for any non-trivial design $\mathbb{Z}_{n}$ is a very difficult computational problem. This explains why the problem of construction of optimal $n$-point designs with smallest covering radius is notoriously difficult (see, for example, recent surveys [16, 17]).

If $r=\mathrm{CR}\left(\mathbb{Z}_{n}\right)$, then $C_{d}\left(\mathbb{Z}_{n}, r\right)$ defined in (1) is equal to 1 , and the whole cube $\mathcal{C}_{d}$ gets covered by the balls. However, we are only interested in reaching the values like 0.9 , when a large part of the ball is covered. There are two main reasons why we are not interested in reaching the value $C_{d}\left(\mathbb{Z}_{n}, r\right)=1$ : (a) practical impossibility of making a numerical checking of the full coverage, if $d$ is large enough, and (b) our approximations lose accuracy when $C_{d}\left(\mathbb{Z}_{n}, r\right)$ closely approaches 1 .

If, for a given $\gamma \in[0,1)$, we have $C_{d}\left(\mathbb{Z}_{n}, r\right) \geq 1-\gamma$, then the corresponding coverage of $\mathcal{C}_{d}$ will be called $(1-\gamma)$-coverage; the corresponding value of $r$ can be called $(1-\gamma)$-covering radius. If $\gamma=0$ then the $(1-\gamma)$-coverage becomes the full coverage and 1-covering radius of $\mathbb{Z}_{n}$ becomes $C_{d}\left(\mathbb{Z}_{n}, r\right)$. Of course, for any $\mathbb{Z}_{n}=\left\{Z_{1}, \ldots, Z_{n}\right\}$ we can reach $C_{d}\left(\mathbb{Z}_{n}, r\right)=1$ by means of increasing $r$. Likewise, for any given $r$ we can reach $C_{d}\left(\mathbb{Z}_{n}, r\right)=1$ by sending $n \rightarrow \infty$. However, we are not interested in very large values of $n$ and try to get the coverage of the most part of the cube $\mathcal{C}_{d}$ with the radius $r$ as small as possible. We will keep in mind the following typical values of $d$ and $n: d=10,20,50 ; n=64,128,512,1024$. Correspondingly, we will illustrate our results in such scenarios.

\subsection{Two Contradictory Criteria and a Compromise}

In choosing $\mathbb{Z}_{n}=\left\{Z_{1}, \ldots, Z_{n}\right\}$, the following two main criteria must be followed:

(i) the volumes of intersections of the cube $\mathcal{C}_{d}$ and each individual ball $\mathcal{B}_{d}\left(Z_{j}, r\right)$ are not very small;

(ii) the volumes of intersections $\mathcal{B}_{d}\left(Z_{j}, r\right) \cap \mathcal{B}_{d}\left(Z_{i}, r\right)$ are small for all $i \neq$ $j(i, j=1, \ldots, n)$. 
These two criteria do not agree with each other. Indeed, as shown in Section 2 (see formulas (11)-(14)), the volume of intersection of the ball $\mathcal{B}_{d}(Z, r)$ and the cube $\mathcal{C}_{d}$ is approximately inversely proportional to $\|Z\|$ and hence criterion (i) favours $Z_{j}$ with small norms. However, if at least some of the points $Z_{j}$ get close to 0 , then the distance between these points gets small and, in view of the formulas of Section A1.7, the volumes of intersections $\mathcal{B}_{d}\left(Z_{j}, r\right) \cap \mathcal{B}_{d}\left(Z_{i}, r\right)$ get large.

This yields that the above two criteria require a compromise in the rule of choosing $\mathbb{Z}_{n}=\left\{Z_{1}, \ldots, Z_{n}\right\}$ as the points $Z_{j}$ should not be too far from 0 but at the same time, not too close. In particular, and this is clearly demonstrated in many examples throughout the paper, the so-called uniformly distributed sequences of points in $\mathcal{C}_{d}$, including 'low-discrepancy sequences' in $\mathcal{C}_{d}$, provide poor covering schemes. This is in a sharp contrast with the asymptotic case $n \rightarrow \infty$ (and hence $r \rightarrow 0$ ), when one of the recommendations (see [2, p. 84]) is to choose $Z_{j}$ 's from a uniformly distributed sequence of points from a set which is slightly larger than $\mathcal{C}_{d}$; this is to facilitate covering of the boundary of $\mathcal{C}_{d}$, as it is much easier to cover the interior of the cube $\mathcal{C}_{d}$ than its boundary.

In our considerations, $n$ is not very large and hence the radius of balls $r$ cannot be small. One of our recommendations for choosing $\mathbb{Z}_{n}=\left\{Z_{1}, \ldots, Z_{n}\right\}$ is to choose $Z_{j}$ 's at random in a cube $\mathcal{C}_{d}(\delta)=[-\delta, \delta]^{d}$ (with $0<\delta \leq 1$ ) with components distributed according to a suitable beta-distribution. The optimal value of $\delta$ is always smaller than 1 and depends on $d$ and $n$. If $d$ is small or $n$ is astronomically large, then the optimal value of $\delta$ could be close to 1 but in most interesting instances this value is significantly smaller than 1 . This implies that the choice $\delta=1$ (for example, if $Z_{j}$ 's form a uniformly distributed sequence of points in the whole cube $\mathcal{C}_{d}$ ) often leads to very poor covering schemes, especially when the dimension $d$ is large (see Tables 1 , 2 and 3 in discussed in Section 3). More generally, we show that for construction of efficient designs $\mathbb{Z}_{n}=\left\{Z_{1}, \ldots, Z_{n}\right\}$, either deterministic or randomized, we have to restrict the norms of the design points $Z_{j}$. We will call this principle ' $\delta$-effect'.

\subsection{Covering a Cube by Smaller Cubes and Quantization}

In Section 4 we consider the problem of $(1-\gamma)$-coverage of the cube $\mathcal{C}_{d}=[-1,1]^{d}$ by smaller cubes (which are $L_{\infty}$-balls). The problem of 1 -covering of cube by cubes has attracted a reasonable attention in mathematical literature (see, e.g., [3, 6]). The problem of $(1-\gamma)$-coverage of a cube by cubes happened to be simpler than the main problem of $(1-\gamma)$-coverage of a cube by Euclidean balls and we have managed to derive closed-form expressions for (a) the volume of intersection of two cubes, and (b) $(1-\gamma)$ coverage, the probability of covering a random point in $\mathcal{C}_{d}$ by $n$ cubes $\mathcal{C}_{d}\left(Z_{i}, r\right)$ for a wide choice of randomized schemes of choosing designs $\mathbb{Z}_{n}=$ $\left\{Z_{1}, \ldots, Z_{n}\right\}$. The results of Section 4 show that the $\delta$-effect holds for the problem of coverage of the cube by smaller cubes in the same degree as for the main problem of Section 3 of covering with balls.

Section 5 is devoted to the following problem of quantization also known as the problem of facility location. Let $X=\left(x_{1}, \ldots, x_{d}\right)$ be uniform on $\mathcal{C}_{d}=[-1,1]^{d}$ and $\mathbb{Z}_{n}=\left\{Z_{1}, \ldots, Z_{n}\right\}$ be an $n$-point design. The mean square quantization error is 
$\theta_{n}=\theta\left(\mathbb{Z}_{n}\right):=\mathbb{E}_{X} \min _{i=1, \ldots, n}\left\|X-Z_{i}\right\|^{2}$. In the case where $Z_{1}, \ldots, Z_{n}$ are i.i.d. uniform on $\mathcal{C}_{d}(\delta)$, we will derive a simple approximation for the expected value of $\theta\left(\mathbb{Z}_{n}\right)$ and clearly demonstrate the $\delta$-effect. Moreover, we will notice a strong similarity between efficient quantization designs and efficient designs constructed in Section 3.

\subsection{Structure of the Paper and Main Results}

In Section 2 we derive accurate approximations for the volume of intersection of an arbitrary $d$-dimensional cube with an arbitrary $d$-dimensional ball. These formulas will be heavily used in Section 3, which is the main section of the paper dealing with the problem of $(1-\gamma)$-coverage of a cube by $n$ balls. In Section 4 we extend some considerations of Section 3 to the problem of $(1-\gamma)$-coverage of the cube $\mathcal{C}_{d}$ by smaller cubes. In Section 5 we argue that there is a strong similarity between efficient quantization designs and efficient designs of Section 3. In Appendix 1, we briefly mention several facts, used in the main part of the paper, related to high-dimensional cubes and balls. In Appendix 2, we prove two simple but very important lemmas about distribution and moments of certain random variables.

Our main contributions in this paper are:

- an accurate approximation (18) for the volume of intersection of an arbitrary $d$-dimensional cube with an arbitrary $d$-dimensional ball;

- an accurate approximation (26) for the expected volume of intersection of the cube $\mathcal{C}_{d}$ with $n$ balls with uniform random centres $Z_{j} \in \mathcal{C}_{d}(\delta)$;

- closed-form expression of Section 4.2 for the expected volume of intersection the cube $\mathcal{C}_{d}$ with $n$ cubes with uniform random centres $Z_{j} \in \mathcal{C}_{d}(\delta)$;

- construction of efficient schemes of quantization and $(1-\gamma)$-coverage of the cube $\mathcal{C}_{d}$ by $n$ balls;

- large-scale numerical study.

We are preparing an accompanying paper [9] in which we will further explore the topics of Sections 3-5 and also consider the problems of quantization and (1 $\gamma)$-coverage in the whole space $\mathbb{R}^{d}$ and the problem of $(1-\gamma)$-coverage of simplices.

\section{Volume of Intersection of a Cube and a Ball}

\subsection{The Main Quantity of Interest}

Consider the following problem. Let us take the cube $\mathcal{C}_{d}=[-1,1]^{d}$ of volume $\operatorname{vol}\left(\mathcal{C}_{d}\right)=2^{d}$ and a ball $\mathcal{B}_{d}(Z, r)=\left\{Y \in \mathbb{R}^{d}:\|Y-Z\| \leq r\right\}$ centred at a point $Z=\left(z_{1}, \ldots, z_{d}\right) \in \mathbb{R}^{d}$; this point $Z$ could be outside $\mathcal{C}_{d}$. Denote the fraction of the cube $\mathcal{C}_{d}$ covered by the ball $\mathcal{B}_{d}(Z, r)$ by

$$
C_{d, Z, r}=\operatorname{vol}\left(\mathcal{C}_{d} \cap \mathcal{B}_{d}(Z, r)\right) / 2^{d} .
$$

Our aim is to approximate $C_{d, Z, r}$ for arbitrary $d, Z$ and $r$. To do this, we shall use CLT (Central Limit Theorem). We will derive a CLT-based normal approximation in 
Section 2.3 and then, using an asymptotic expansion in the CLT for non-identically distributed r.v., we will improve this normal approximation in Section 2.4. In Section A1.8 we consider a more direct approach for approximating $C_{d, Z, r}$ based on the use of characteristic functions and the fact that $C_{d, Z, r}$ is a c.d.f. of $\|U-Z\|$, where $U=\left(u_{1}, \ldots, u_{d}\right)$ is random vector with uniform distribution on $\mathcal{C}_{d}$. From this, $C_{d, Z, r}$ can be expressed through the convolution of one-dimensional c.d.f's. Using this approach we can evaluate the quantity $C_{d, Z, r}$ with high accuracy but the calculations are rather time-consuming. Moreover, entirely new computations have to be made for different $Z$ and, therefore, we much prefer the approximation of Section 2.4.

Note that in the special case $Z=0$, several approximations for the quantity $C_{d, 0, r}$ have been derived in [15] but their methods cannot be generalized to arbitrary $Z$.

\subsection{A Generalization of the Quantity (3)}

In the next sections, we will need another quantity which slightly generalizes (3). Assume that we have the cube $\mathcal{C}_{d}(\delta)=[-\delta, \delta]^{d}$ of volume $\operatorname{vol}\left(\mathcal{C}_{d}(\delta)\right)=(2 \delta)^{d}$, the ball $\mathcal{B}_{d}\left(Z^{\prime}, r^{\prime}\right)=\left\{Y \in \mathbb{R}^{d}:\left\|Y-Z^{\prime}\right\| \leq r^{\prime}\right\}$ with a centre at a point $Z^{\prime}=$ $\left(z_{1}^{\prime}, \ldots, z_{d}^{\prime}\right)$. Denote the fraction of the cube $\mathcal{C}_{d}(\delta)$ covered by the ball $\mathcal{B}_{d}\left(Z^{\prime}, r^{\prime}\right)$ by

$$
\left.C_{d, Z^{\prime}, r^{\prime}}^{(\delta)}=\operatorname{vol}\left(\mathcal{C}_{d}(\delta) \cap \mathcal{B}_{d\left(Z^{\prime}\right.}, r^{\prime}\right)\right) /(2 \delta)^{d} .
$$

Then, by changing the coordinates and the radius

$$
Z=Z^{\prime} / \delta=\left(z_{1}^{\prime} / \delta, \ldots, z_{d}^{\prime} / \delta\right) \text { and } r=r^{\prime} / \delta,
$$

we obtain

$$
C_{d, Z^{\prime}, r^{\prime}}^{(\delta)}=C_{d, Z, r}
$$

\subsection{Normal Approximation for the Quantity (3)}

Let $U=\left(u_{1}, \ldots, u_{d}\right)$ be a random vector with uniform distribution on $\mathcal{C}_{d}$ so that $u_{1}, \ldots, u_{d}$ are i.i.d.r.v. uniformly distributed on $[-1,1]$. Then for given $Z=$ $\left(z_{1}, \ldots, z_{d}\right) \in \mathbb{R}^{d}$ and any $r>0$,

$$
C_{d, Z, r}=\mathbb{P}\{\|U-Z\| \leq r\}=\mathbb{P}\left\{\|U-Z\|^{2} \leq r^{2}\right\}=\mathbb{P}\left\{\sum_{j=1}^{d}\left(u_{j}-z_{j}\right)^{2} \leq r^{2}\right\} .
$$

That is, $C_{d, Z, r}$, as a function of $r$, is the c.d.f. of the r.v. $\|U-Z\|$.

Let $u$ have a uniform distribution on $[-1,1]$ and $|z| \leq 1$. In view of Lemma 1 of Appendix 2, the density of the r.v. $\eta_{z}=(u-z)^{2}$ is

$$
\varphi_{z(t)}= \begin{cases}1 /(2 \sqrt{t}) & \text { for } 0<t \leq(1-|z|)^{2} \\ 1 /(4 \sqrt{t}) & \text { for }(1-|z|)^{2}<t \leq(1+|z|)^{2} \\ 0 & \text { otherwise }\end{cases}
$$

and

$$
\mathbb{E} \eta_{z}=z^{2}+\frac{1}{3}, \quad \operatorname{var}\left(\eta_{z}\right)=\frac{4}{3}\left(z^{2}+\frac{1}{15}\right), \quad \mu_{z}^{(3)}=\frac{16}{15}\left(z^{2}+\frac{1}{63}\right),
$$


where $\mu_{z}^{(3)}$ is the third central moment: $\mu_{z}^{(3)}=E\left[\eta_{z}-E \eta_{z}\right]^{3}$.

For $|z|>1$, the density of $\eta_{z}=(u-z)^{2}$ is

$$
\varphi_{z(t)}= \begin{cases}1 /(4 \sqrt{t}) & \text { for }(1-|z|)^{2}<t \leq(1+|z|)^{2} \\ 0 & \text { otherwise }\end{cases}
$$

with expressions (8) for $\mathbb{E} \eta_{z}, \operatorname{var}\left(\eta_{z}\right)$ and $\mu_{z}^{(3)}$ not changing.

Consider the r.v.

$$
\|U-Z\|^{2}=\sum_{i=1}^{d} \eta_{z_{j}}=\sum_{j=1}^{d}\left(u_{j}-z_{j}\right)^{2}
$$

From (8), its mean is

$$
\mu_{d, Z}=\mathbb{E}\|U-Z\|^{2}=\|Z\|^{2}+\frac{d}{3} .
$$

Using independence of $u_{1}, \ldots, u_{d}$, we also obtain from (8):

$$
\sigma_{d, Z}^{2}=\operatorname{var}\left(\|U-Z\|^{2}\right)=\frac{4}{3}\left(\|Z\|^{2}+\frac{d}{15}\right)
$$

and

$$
\mu_{d, Z}^{(3)}=\mathbb{E}\left[\|U-Z\|^{2}-\mu_{d, Z}\right]^{3}=\sum_{j=1}^{d} \mu_{z_{j}}^{(3)}=\frac{16}{15}\left(\|Z\|^{2}+\frac{d}{63}\right) .
$$

If $d$ is large enough then the conditions of the CLT for $\|U-Z\|^{2}$ are approximately met and the distribution of $\|U-Z\|^{2}$ is approximately normal with mean $\mu_{d, Z}$ and variance $\sigma_{d, Z}^{2}$. That is, we can approximate $C_{d, Z, r}$ by

$$
C_{d, Z, r} \cong \Phi\left(\frac{r^{2}-\mu_{d, Z}}{\sigma_{d, Z}}\right),
$$

where $\Phi(\cdot)$ is the c.d.f. of the standard normal distribution:

$$
\Phi(t)=\int_{-\infty}^{t} \varphi(v) d v \text { with } \varphi(v)=\frac{1}{\sqrt{2 \pi}} e^{-v^{2} / 2} .
$$

The approximation (14) has acceptable accuracy if $C_{d, Z, r}$ is not very small; for example, it falls inside a $2 \sigma$-confidence interval generated by the standard normal distribution (see Figs. $1 \mathrm{a}$ and $1 \mathrm{~b}$ as examples). Let $p_{\beta}$ be the quantile of the standard normal distribution defined by $\Phi(\beta)=1-p_{\beta}$; for example, $p_{\beta} \simeq 0.05$ for $\beta=2$. As follows from (11), (12) and the approximation (14), we expect the approximate inequality $C_{d, Z, r} \gtrsim p_{\beta}$ if

$$
r \geq R_{d,\|Z\|, \beta}=\left[\|Z\|^{2}+d / 3-2 \beta \sqrt{\|Z\|^{2} / 3+d / 45}\right]^{1 / 2} .
$$


a

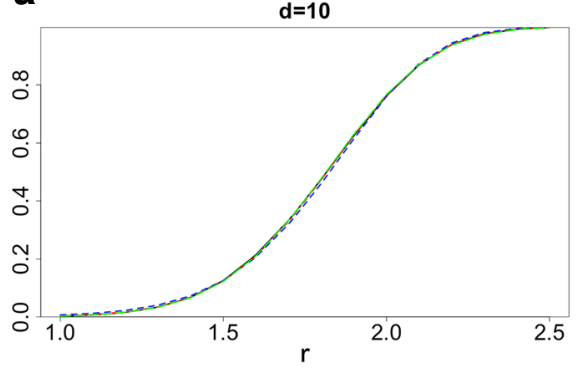

b

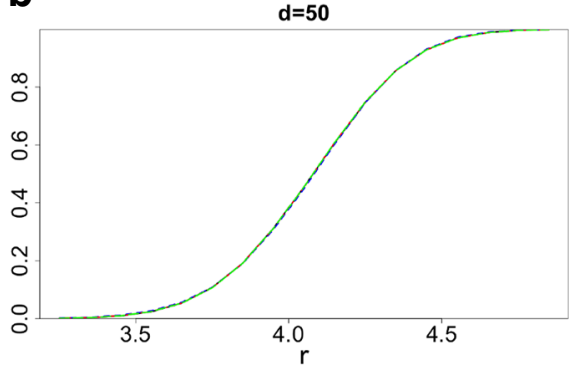

Fig. 1 (a) $d=10, Z=0, r \in[1,2.5]$, (b) $d=50, Z=0, r \in[3.2,4.9]$

In many cases discussed in Section 3, the radius $r$ does not satisfy the inequality (15) with $\beta=2$ and even $\beta=3$ and hence the normal approximation (14) is not satisfactorily accurate; this can be evidenced from Figs. 1a, 1b, 2a, 2b, 3a, 3b, 4a, 4b, 5a, 5b, $6 \mathrm{a}, 6 \mathrm{~b}, 7 \mathrm{a}, 7 \mathrm{~b}, 8 \mathrm{a}$ and $8 \mathrm{~b}$.

In the next section, we improve the approximation (14) by using an Edgeworthtype expansion in the CLT for sums of independent non-identically distributed r.v.

\subsection{Improved Normal Approximation}

General expansion in the central limit theorem for sums of independent non-identical r.v. has been derived by V. Petrov (see Theorem 7 in Chapter 6 in [10]; see also Proposition 1.5.7 in [12]). The first three terms of this expansion have been specialized by V. Petrov in Section 5.6 in [11]. By using only the first term in this expansion, we obtain the following approximation for the distribution function of $\|U-Z\|^{2}$ :

$$
P\left(\frac{\|U-Z\|^{2}-\mu_{d, Z}}{\sigma_{d, Z}} \leq x\right) \cong \Phi(x)+\frac{\mu_{d, Z}^{(3)}}{6\left(\sigma_{d, Z}^{2}\right)^{3 / 2}}\left(1-x^{2}\right) \varphi(x),
$$

a

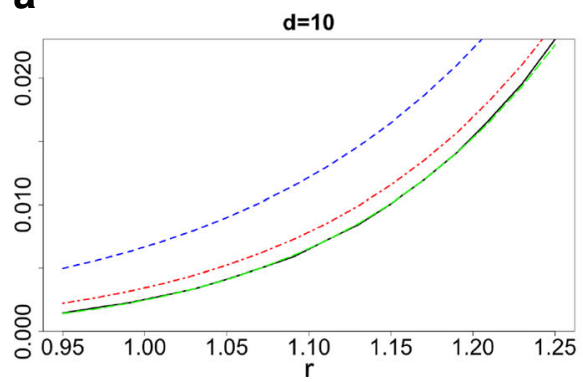

b

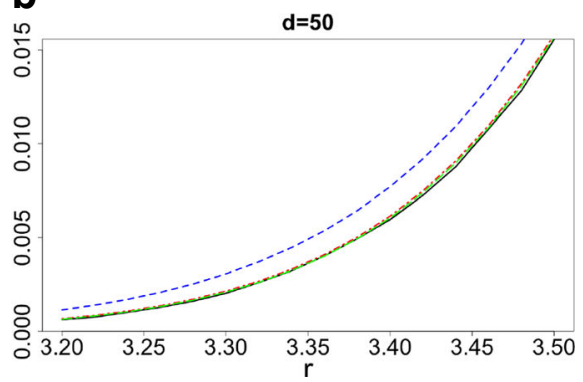

Fig. 2 (a) $d=10, Z=0, r \in[0.95,1.25]$, (b) $d=50, Z=0, r \in[3.2,3.5]$ 

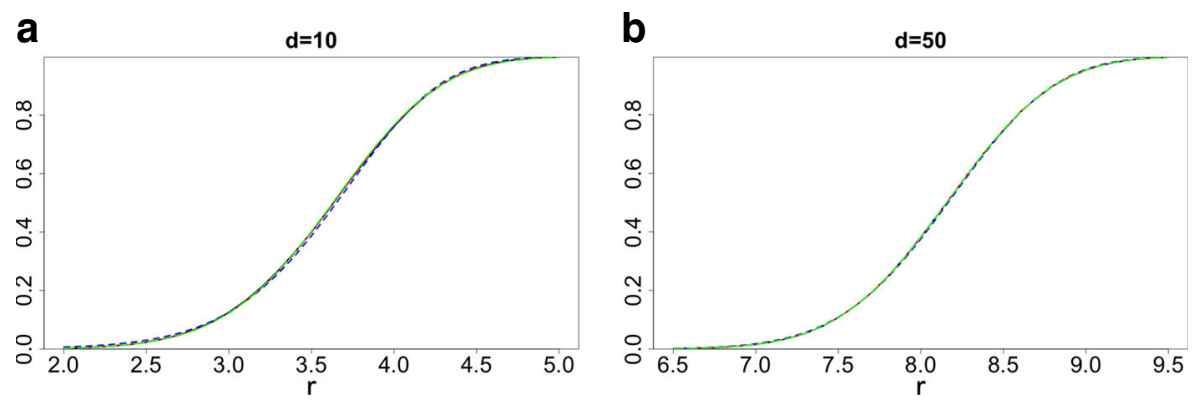

Fig. 3 (a) $d=10, Z$ is a vertex of $\mathcal{C}_{d}, r \in[2,5],(\mathbf{b}) d=50, Z$ is a vertex of $\mathcal{C}_{d}, r \in[6.5,9.5]$
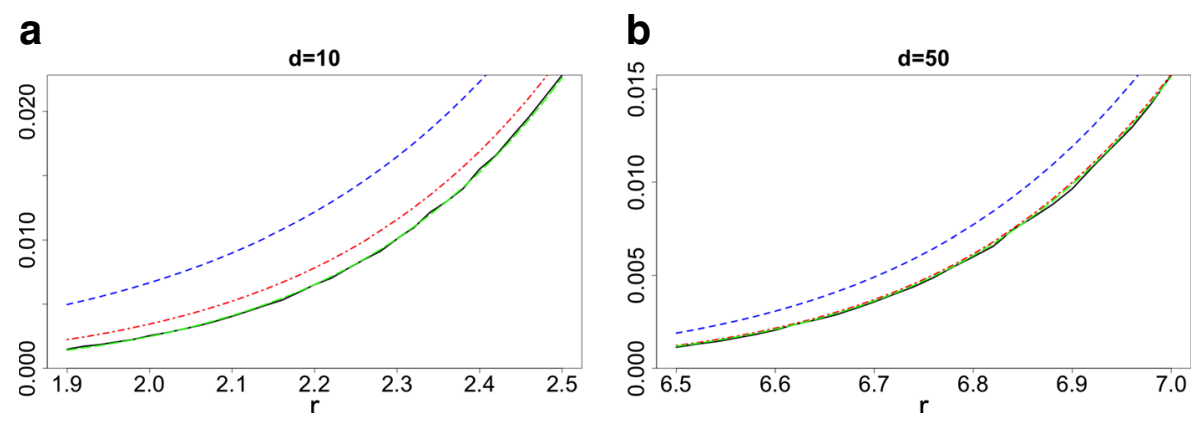

Fig. 4 (a) $d=10, Z$ is a vertex of $\mathcal{C}_{d}, r \in[1.9,2.5],(\mathbf{b}) d=50, Z$ is a vertex of $\mathcal{C}_{d}, r \in[6.5,7]$
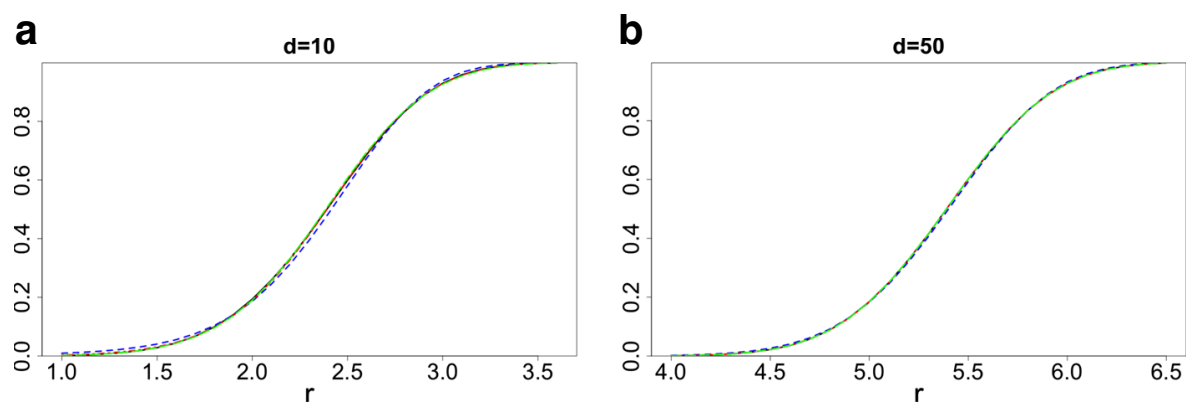

Fig. 5 (a) $Z$ is at half-diagonal with $\|Z\|=\frac{1}{2} \sqrt{10}$, (b) $Z$ is at half-diagonal, $\|Z\|=\frac{1}{2} \sqrt{50}$ 

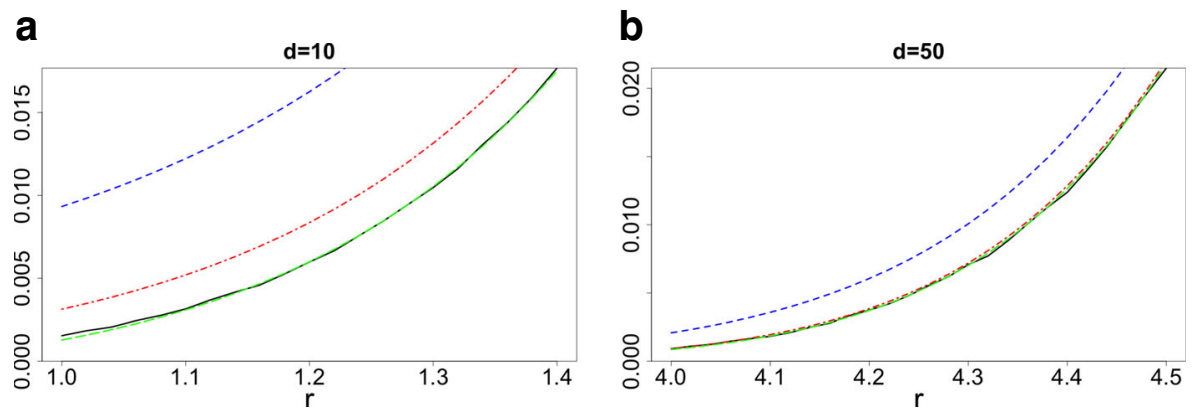

Fig. 6 (a) $Z$ is at half-diagonal, $\|Z\|=\frac{1}{2} \sqrt{10}$, (b) $Z$ is at half-diagonal, $\|Z\|=\frac{1}{2} \sqrt{50}$ )
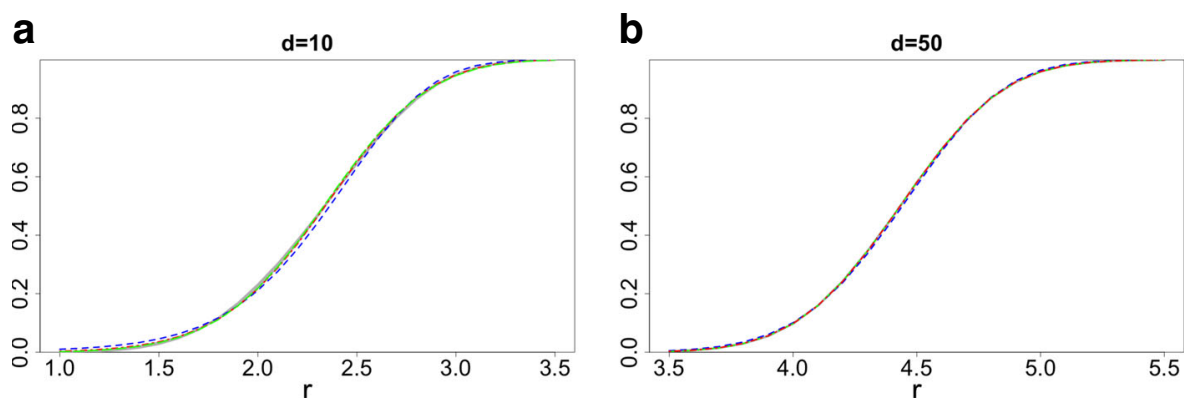

Fig. 7 (a) $d=10, Z \in \mathcal{S}_{10}(0,1.5), r \in[1,3.5]$, (b) $d=50, Z \in \mathcal{S}_{50}(0,1.75), r \in[3.5,5.5]$
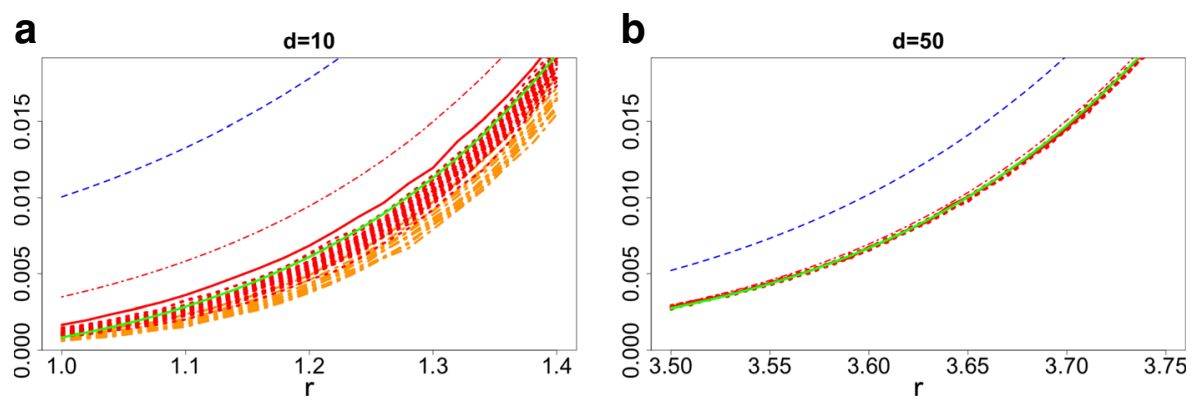

Fig. 8 (a) $d=10, Z \in \mathcal{S}_{10}(0,1.5), r \in[1,1.4]$, (b) $d=50, Z \in \mathcal{S}_{50}(0,1.75), r \in[3.5,3.75]$ 
leading to the following improved form of (14):

$$
C_{d, Z, r} \cong \Phi(t)+\frac{\|Z\|^{2}+d / 63}{5 \sqrt{3}\left(\|Z\|^{2}+d / 15\right)^{3 / 2}}\left(1-t^{2}\right) \varphi(t),
$$

where

$$
t=t_{d,\|Z\|, r}=\frac{r^{2}-\mu_{d, Z}}{\sigma_{d, Z}}=\frac{\sqrt{3}\left(r^{2}-\|Z\|^{2}-d / 3\right)}{2 \sqrt{\|Z\|^{2}+d / 15}} .
$$

From the viewpoint of Section 3, the range of most important values of $t$ from (17) is $-3 \pm 1$. For such values of $t$, the uncorrected normal approximation (14) significantly overestimates the values of $C_{d, Z, r}$ (see Figs. 1a, 1b, 2a, 2b, 3a, 3b, 4a, 4b, 5a, 5b, 6a, 6b, 7a, 7b, 8a and 8b below). The approximation (16) brings the normal approximation down and makes it much more accurate. The other terms in Petrov's expansion of [10] and [11] continue to bring the approximation down (in a much slower fashion) so that the approximation (16) still slightly overestimates the true value of $C_{d, Z, r}$ (at least, in the range of interesting values of $t$ from (17)). However, if $d$ is large enough (say, $d \geq 20$ ) then the approximation (16) is very accurate and no further correction is needed.

A very attractive feature of the approximations (14) and (17) is their dependence on $Z$ through $\|Z\|$ only. We could have specialized for our case the next terms in Petrov's approximation but these terms no longer depend on $\|Z\|$ only (this fact can be verified from the formula (46) for the fourth moment of the r.v. $\left.v_{z}=(z-u)^{2}\right)$ and hence the next terms are much more complicated. Moreover, adding one or two extra terms from Petrov's expansion to the approximation (16) does not fix the problem entirely for all $Z$ and $r$. Instead, we propose a slight adjustment to the r.h.s of (16) to improve this approximation, especially for small dimensions. Specifically, we suggest the approximation

$$
C_{d, Z, r} \cong \Phi(t)+c_{d} \frac{\|Z\|^{2}+d / 63}{5 \sqrt{3}\left(\|Z\|^{2}+d / 15\right)^{3 / 2}}\left(1-t^{2}\right) \varphi(t),
$$

where $c_{d}=1+3 / d$ if the point $Z$ lies on the diagonal of the cube $\mathcal{C}_{d}$ and $c_{d}=1+4 / d$ for a typical (random) point $Z$. For typical (random) points $Z \in \mathcal{C}_{d}$, the values of $C_{d, Z, r}$ are marginally smaller than for the points on the diagonal of $\mathcal{C}_{d}$ having the same norm, but the difference is very small. In addition to the points on the diagonal, there are other special points: the points whose components are all zero except for one. For such points, the values of $C_{d, Z, r}$ are smaller than for typical points $Z$ with the same norm, especially for small $r$. Such points, however, are of no value for us as they are not typical and we have never observed in simulations random points that come close to these truly exceptional points.

\subsection{Simulation Study}

In Figs.1a, 1b, 2a, 2b, 3a, 3b, 4a, 4b, 5a, 5b, 6a, 6b, 7a, 7b, 8a and 8b we demonstrate the accuracy of approximations (14), (16) and (18) for $C_{d, Z, r}$ in dimensions $d=$ 10, 50 for the following locations of $Z$ :

(i) $Z=0$, the centre of the cube $\mathcal{C}_{d}$; 
(ii) $\|Z\|=\sqrt{d}$, with $Z$ being a vertex of the cube $\mathcal{C}_{d}$;

(iii) $Z$ lies on a diagonal of $\mathcal{C}_{d}$ with $\left|z_{j}\right|=\lambda \geq 0$ for all $j=1, \ldots, d$ and $\|Z\|=\lambda \sqrt{d}$

(iv) $Z$ is a random vector uniformly distributed on the sphere $\mathcal{S}_{d}(0, v)$ with some $v>0$.

There are figures of two types. In the figures of the first type, we plot $C_{d, Z, r}$ over a wide range of $r$ ensuring that values of $C_{d, Z, r}$ lie in the whole range $[0,1]$. In the figures of the second type, we plot $C_{d, Z, r}$ over a much smaller range of $r$ with $C_{d, Z, r}$ lying in the range $[0, \varepsilon]$ for some small positive $\varepsilon$ such as $\varepsilon=0.015$. For the purpose of using the approximations of Section 3, we need to assess the accuracy of all approximations for smaller values of $C_{d, Z, r}$ and hence the second type of plots are often more insightful. In Figs. 1a, 1b, 2a, 2b, 3a, 3b, 4a, 4b, 5a, 5b, 6a, 6b, 7a and 7b the solid black line depicts values of $C_{d, Z, r}$ computed via Monte Carlo methods, the blue dashed, the red dot-dashed and green long dashed lines display approximations (14), (16) and (18), respectively.

In the case where $Z$ is a random vector uniformly distributed on a sphere $\mathcal{S}_{d}(0, v)$, the style of the figures of the second type is slightly changed to adapt for this choice of $Z$ and provide more information for $Z$ which do or do not belong to the cube $\mathcal{C}_{d}$. In Figs. $8 \mathrm{a}$ and $8 \mathrm{~b}$, the thick dashed red lines correspond to random points $Z \in$ $\mathcal{S}_{d}(0, v) \cap \mathcal{C}_{d}$. The thick dot-dashed orange lines correspond to random points $Z \in$ $\mathcal{S}_{d}(0, v)$ such that $Z \notin \mathcal{C}_{d}$. Approximations (14) and (16) are depicted in the same manner as previous figures but the approximation (18) is now represented by a solid green line. The thick solid red line displays values of $C_{d, Z, r}$ for $Z$ on the diagonal of $\mathcal{C}_{d}$ with $\|Z\|=v$ with $v=1.5$ for $d=10$ and $v=1.75$ for $d=50$.

From the simulations that led to Figs. 1a, 1b, 2a, 2b, 3a, 3b, 4a, 4b, 5a, 5b, 6a, 6b, $7 \mathrm{a}, 7 \mathrm{~b}, 8 \mathrm{a}$ and $8 \mathrm{~b}$ we can make the following conclusions.

- The normal approximation (14) is quite satisfactory unless the value $C_{d, Z, r}$ is small.

- The accuracy of all approximations improves as $d$ grows.

- The approximation (18) is very accurate even if the values $C_{d, Z, r}$ are very small.

- If $d$ is large enough then the approximations (16) and (18) are practically identical and are extremely accurate.

\section{Covering a Cube by $n$ Balls}

In this section, we consider the main problem of covering the cube $\mathcal{C}_{d}=[-1,1]^{d}$ by the union of $n$ balls $\mathcal{B}_{d}\left(Z_{j}, r\right)$ as formulated in Section 1.2. We will discuss different schemes of choosing the set of ball centres $\mathbb{Z}_{n}=\left\{Z_{1}, \ldots, Z_{n}\right\}$ for given $d$ and $n$. The radius $r$ will then be chosen to achieve the required probability of covering: $C_{d}\left(\mathbb{Z}_{n}, r\right) \geq 1-\gamma$. Most of the schemes will involve one or several parameters which we will want to choose in an optimal way. 


\subsection{The Main Covering Scheme}

The following will be our main scheme for choosing $\mathbb{Z}_{n}=\left\{Z_{1}, \ldots, Z_{n}\right\}$.

Scheme 1. $Z_{1}, \ldots, Z_{n}$ are i.i.d. random vectors uniformly distributed in the cube $\mathcal{C}_{d}(\delta)=[-\delta, \delta]^{d}$, where $\delta \in[0,1]$ is a parameter

We will formulate several other covering schemes and compare them with Scheme 1. The reasons why we have chosen Scheme 1 as the main scheme are the following.

- It is easier to theoretically investigate than all other non-trivial schemes.

- It includes, as a special case when $\delta=1$, the scheme which is very popular in practice of Monte Carlo [8] and global random search [18, 19] and is believed to be rather efficient (this is not true).

- Numerical studies provided below show that Scheme 1 with optimal $\delta$ provides coverings which are rather efficient, especially for large $d$ (see Section 3.5 for a discussion regarding this issue).

\subsection{Theoretical Investigation of Scheme 1}

Let $Z_{1}, \ldots, Z_{n}$ be i.i.d. random vectors uniformly distributed in the cube $\mathcal{C}_{d}(\delta)$ with $0<\delta \leq 1$. Then, for given $U=\left(u_{1}, \ldots, u_{d}\right) \in \mathbb{R}^{d}$,

$$
\begin{aligned}
\mathbb{P}\left\{U \in \mathcal{B}_{d}\left(\mathbb{Z}_{n}, r\right)\right\} & =1-\prod_{j=1}^{n} \mathbb{P}\left\{U \notin \mathcal{B}_{d}\left(Z_{j}, r\right)\right\} \\
& =1-\prod_{j=1}^{n}\left(1-\mathbb{P}\left\{U \in \mathcal{B}_{d}\left(Z_{j}, r\right)\right\}\right) \\
& =1-\left(1-\mathbb{P}_{Z}\{\|U-Z\| \leq r\}\right)^{n}
\end{aligned}
$$

where $\mathcal{B}_{d}\left(\mathbb{Z}_{n}, r\right)$ is defined in (2). The main characteristic of interest $C_{d}\left(\mathbb{Z}_{n}, r\right)$, defined in (1), the proportion of the cube covered by the union of balls $\mathcal{B}_{d}\left(\mathbb{Z}_{n}, r\right)$, is simply

$$
C_{d}\left(\mathbb{Z}_{n}, r\right)=\mathbb{E}_{U} \mathbb{P}\left\{U \in \mathcal{B}_{d}\left(\mathbb{Z}_{n}, r\right)\right\}
$$

Continuing (19), note that

$$
\mathbb{P}_{Z}\{\|U-Z\| \leq r\}=\mathbb{P}_{Z}\left\{\sum_{j=1}^{d}\left(z_{j}-u_{j}\right)^{2} \leq r^{2}\right\}=C_{d, U, r}^{(\delta)},
$$

where $C_{d, U, r}^{(\delta)}$ is defined by the formula (4). From (5) and 6 we have $C_{d, U, r}^{(\delta)}=$ $C_{d, U / \delta, r / \delta}$ where $C_{d, U / \delta, r / \delta}$ is the quantity defined by (3). This quantity can be approximated in a number of different ways as shown in Section 2. We will compare (14), the simplest of the approximations, with the approximation given in (18). 
Approximation (14) gives

$$
C_{d, U, r}^{(\delta)}=C_{d, U / \delta, r / \delta} \cong \Phi\left(\frac{(r / \delta)^{2}-\|U\|^{2} / \delta^{2}-d / 3}{2 \sqrt{\|U\|^{2} /\left(3 \delta^{2}\right)+d / 45}}\right),
$$

whereas approximation (18) provides

$$
C_{d, U, r}^{(\delta)} \cong \Phi\left(t_{\delta}\right)+c_{d} \frac{\|U\|^{2} / \delta^{2}+d / 63}{5 \sqrt{3}\left(\|U\|^{2} / \delta^{2}+d / 15\right)^{3 / 2}}\left(1-t_{\delta}^{2}\right) \varphi\left(t_{\delta}\right),
$$

with $c_{d}=1+4 / d$ and

$$
t_{\delta}=\frac{(r / \delta)^{2}-\|U\|^{2} / \delta^{2}-d / 3}{2 \sqrt{\|U\|^{2} /\left(3 \delta^{2}\right)+d / 45}} .
$$

From (37), $\mathbb{E}\|U\|^{2}=d / 3$ and $\operatorname{var}\left(\|U\|^{2}\right)=4 d / 45$. Moreover, if $d$ is large enough then $\|U\|^{2}=\sum_{j=1}^{d} u_{j}^{2}$ is approximately normal.

We shall simplify the expression (19) by using the approximation

$$
(1-t)^{n} \simeq e^{-n t}
$$

which is a good approximation for small values of $t$ and moderate values of $n t$; this agrees with the ranges of $d, n$ and $r$ we are interested in.

We can combine the expressions (20) and (19) with approximations (22), (23) and (24) as well as with the normal approximation for the distribution of $\|U\|^{2}$, to arrive at two final approximations for $C_{d}\left(\mathbb{Z}_{n}, r\right)$ that differ in complexity. If the original normal approximation of (22) is used then we obtain

$$
C_{d}\left(\mathbb{Z}_{n}, r\right) \simeq 1-\int_{-\infty}^{\infty} \psi_{1}(s) \varphi(s) d s
$$

with

$$
\psi_{1}(s)=\exp \left\{-n \Phi\left(c_{s}\right)\right\}, \quad c_{s}=\frac{3(r / \delta)^{2}-s^{\prime}-d}{2 \sqrt{s^{\prime}+d / 5}}, \quad s^{\prime}=(d+2 s \sqrt{d / 5}) / \delta^{2} .
$$

If approximation (23) is used, we obtain:

$$
C_{d}\left(\mathbb{Z}_{n}, r\right) \simeq 1-\int_{-\infty}^{\infty} \psi_{2}(s) \varphi(s) d s
$$

with

$$
\psi_{2}(s)=\exp \left\{-n\left(\Phi\left(c_{s}\right)+\left(1+\frac{4}{d}\right) \frac{s^{\prime}+d / 21}{5\left[s^{\prime}+d / 5\right]^{3 / 2}}\left(1-c_{s}^{2}\right) \varphi\left(c_{s}\right)\right)\right\} .
$$

\subsection{Simulation Study for Assessing Accuracy of Approximations (25) and (26)}

In Figs. 9a, 9b, 10a, 10b, 11a and $11 \mathrm{~b}, C_{d}\left(\mathbb{Z}_{n}, r\right)$ is represented by a solid black line and has been obtained via Monte Carlo methods. Approximation (25) is indicated by a dashed blue line and approximation (26) is represented by long dashed green lines. All figures demonstrate that approximation (26) is extremely accurate across different dimensions and values of $n$. This approximation is much superior to approximation (25). 

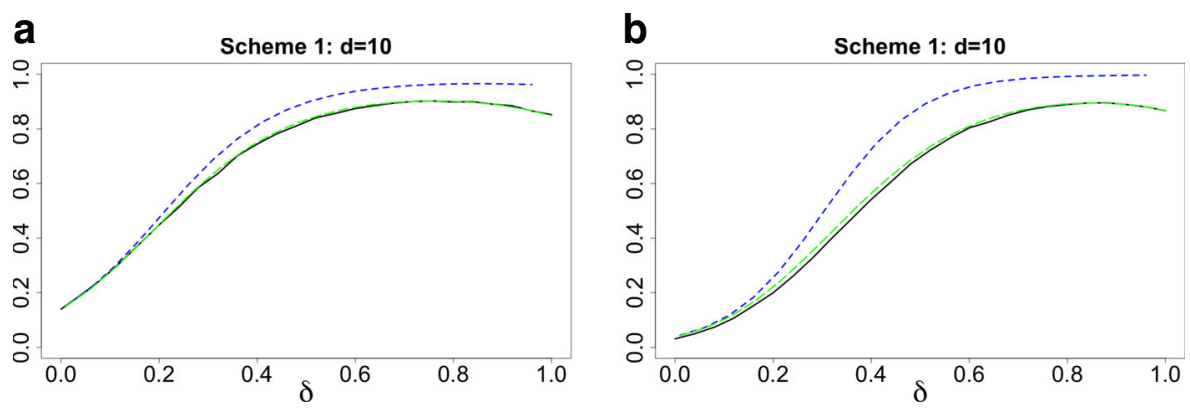

Fig. 9 (a) $C_{d}\left(\mathbb{Z}_{n}, r\right)$ and approximations: $n=128$, (b) $C_{d}\left(\mathbb{Z}_{n}, r\right)$ and approximations: $n=512$
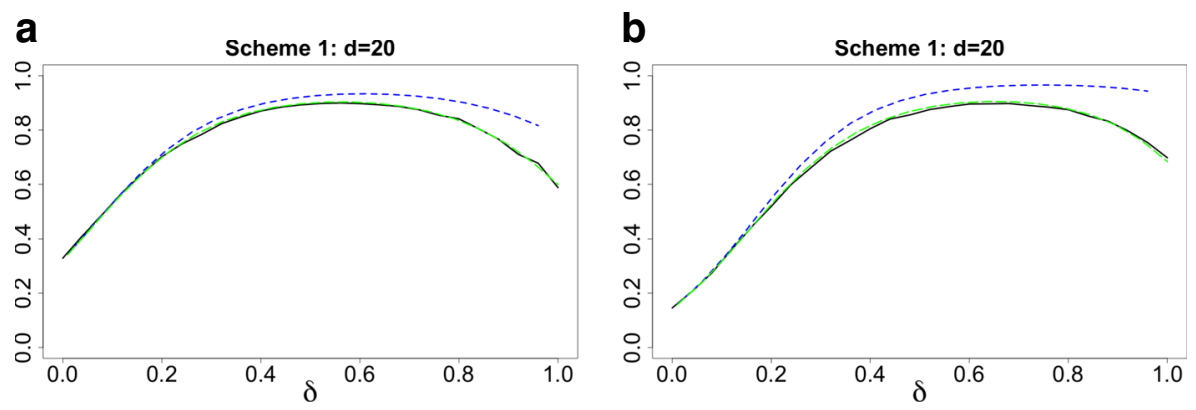

Fig. 10 (a) $C_{d}\left(\mathbb{Z}_{n}, r\right)$ and approximations: $n 128$, (b) $C_{d}\left(\mathbb{Z}_{n}, r\right)$ andapproximations: $n=512$
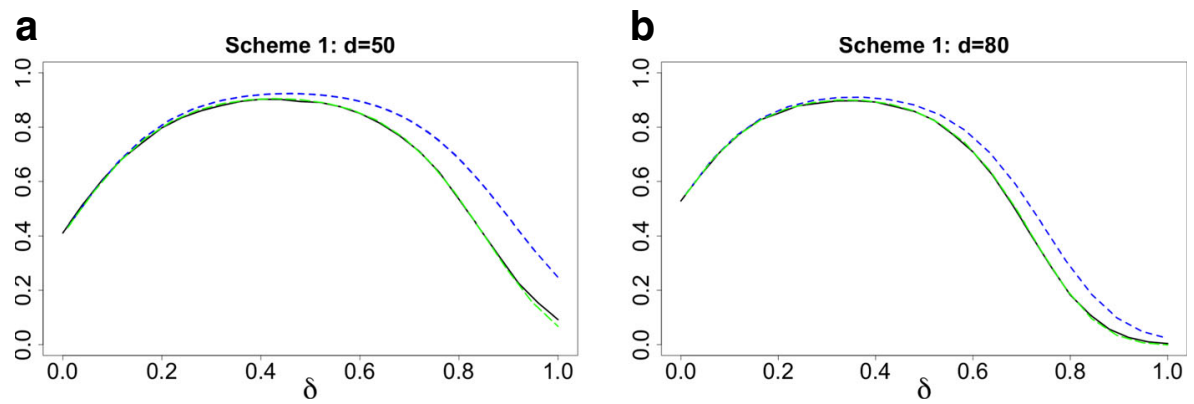

Fig. 11 (a) $C_{d}\left(\mathbb{Z}_{n}, r\right)$ and approximations: $n=512$, (b) $C_{d}\left(\mathbb{Z}_{n}, r\right)$ and approximations: $n=512$ 


\subsection{Other Schemes}

In addition to Scheme 1, we have also considered the following schemes for choosing $\mathbb{Z}_{n}=\left\{Z_{1}, \ldots, Z_{n}\right\}$.

Scheme 2. $Z_{1}=0 ; Z_{2}, \ldots, Z_{n}$ are i.i.d. random vectors uniformly distributed in the cube $\mathcal{C}_{d}(\delta)=[-\delta, \delta]^{d}$

Scheme 3. $Z_{1}, \ldots, Z_{n}$ are taken from the minimum-aberration fractional factorial design on vertices of the cube $\mathcal{C}_{d}(\delta)=[-\delta, \delta]^{d}$

Scheme 4. $Z_{1}, \ldots, Z_{n}$ are i.i.d. random vectors on $\mathcal{C}_{d}(\delta)$ with independent components distributed according to beta-distribution with density (34) with some $\alpha>0$

Scheme 5. $Z_{1}, \ldots, Z_{n}$ are i.i.d. random vectors uniformly distributed in the ball $\mathcal{B}_{d}(\delta)$

Scheme 6. $Z_{1}, \ldots, Z_{n}$ are i.i.d. random vectors uniformly distributed on the sphere $\mathcal{S}_{d}(\delta)$

Scheme 7. $Z_{1}, \ldots, Z_{n}$ are taken from a low-discrepancy Sobol's sequence on the cube $\mathcal{C}_{d}(\delta)$

The rationale behind the choice of these schemes is as follows. By studying Scheme 2, we test the importance of inclusion of 0 into $\mathbb{Z}_{n}$. We propositioned that if we included 0 into $\mathbb{Z}_{n}$, the optimal value of $\delta$ may increase for some of the schemes making them more efficient; this effect has not been detected.

Scheme 3 with optimal $\delta$ is an obvious candidate for being the most efficient. Unlike all other schemes considered, Scheme 3 is only defined for the values of $n$ of the form $n=2^{k}$ with $k \leq d$.

By using Scheme 4, we test the possibility of improving Scheme 1 by changing the distribution of points in the cube $\mathcal{C}_{d}(\delta)$. We have found that the effect of distribution is strong and smaller values of $\alpha$ lead to more efficient covering schemes. By choosing $\alpha$ small enough, like $\alpha=0.1$, we achieve the average efficiency of the covering schemes which is rather close to the efficiency of Scheme 3. Tables 1, 2 and 3 contain results obtained for Scheme 4 with $\alpha=0.5$ and $\alpha=1.5$; if $\alpha=1$ then Scheme 4 becomes Scheme 1.

From Section A1.4, we know that for constructing efficient designs we have to somehow restrict the norms of $Z_{j}$ 's. In Schemes 5 and 6, we are trying to do this in an alternative way to Schemes 1 and 4 .

Scheme 7 is a natural improvement of Scheme 1 . As a particular case with $\delta=1$, it contains one of the best known low-discrepancy sequences and hence Scheme 7 with $\delta=1$ serves as the main benchmark with which we compare other schemes. For construction, we have used the R-implementation of the Sobol's sequences; it is based on [4].

For all the schemes excluding Scheme 3 , the sequences $\mathbb{Z}_{n}=\left\{Z_{1}, \ldots, Z_{n}\right\}$ are nested so that $\mathbb{Z}_{n} \subset \mathbb{Z}_{m}$ for all $n<m$; using the terminology of [6], these schemes provide on-line coverings of the cube. Note that for the chosen values of $n$, Scheme 7 also has some advantage over other schemes considered. Indeed, despite Sobol's sequences are nested, the values $n$ of the form $n=2^{k}$ are special for the Sobol's 
Table 1 Values of $r$ and $\delta$ (in parentheses) to achieve 0.9 coverage for $d=10$

\begin{tabular}{lllll}
\hline$d=10$ & & & & \\
& $n=64$ & $n=128$ & $n=512$ & $n=1024$ \\
\hline Scheme 1 & $1.632(0.70)$ & $1.520(0.78)$ & $1.291(0.86)$ & $1.195(0.90)$ \\
Scheme 1, $\delta=1$ & $1.720(1.00)$ & $1.577(1.00)$ & $1.319(1.00)$ & $1.215(1.00)$ \\
Scheme 2 & $1.634(0.70)$ & $1.520(0.78)$ & $1.291(0.86)$ & $1.195(0.90)$ \\
Scheme 3 & $1.530(0.44)$ & $1.395(0.48)$ & $1.115(0.50)$ & $1.075(0.50)$ \\
Scheme 4, $\alpha=0.5$ & $1.629(0.58)$ & $1.505(0.65)$ & $1.270(0.72)$ & $1.165(0.75)$ \\
Scheme 4, $\alpha=1.5$ & $1.635(0.80)$ & $1.525(0.88)$ & $1.310(1.00)$ & $1.210(1.00)$ \\
Scheme 5 & $1.645(1.40)$ & $1.530(1.50)$ & $1.330(1.75)$ & $1.250(1.75)$ \\
Scheme 6 & $1.642(1.25)$ & $1.532(1.35)$ & $1.330(1.50)$ & $1.250(1.70)$ \\
Scheme 7 & $1.595(0.72)$ & $1.485(0.80)$ & $1.280(0.85)$ & $1.170(0.88)$ \\
Scheme 7, $\delta=1$ & $1.678(1.00)$ & $1.534(1.00)$ & $1.305(1.00)$ & $1.187(1.00)$ \\
\hline
\end{tabular}

sequences and for such values of $n$ the Sobol's sequences possess extra uniformity properties that they do not possess for other values of $n$.

\subsection{Numerical Comparison of Schemes}

In Tables 1, 2 and 3, for Schemes 1, 2, 4, 5 and 6, we present the smallest values of $r$ required to achieve an 0.9 -coverage on average. For these schemes, the value inside the parentheses shows the average value of $\delta$ required to obtain 0.9 -coverage. For Schemes 3 and 7, we give the smallest value of $r$ needed for a 0.9 -coverage. For these two schemes, the value within the bracket corresponds to the (non random) value of $\delta$ with which we attain such a coverage.

Table 2 Values of $r$ and $\delta$ (in parentheses) to achieve 0.9 coverage for $d=20$

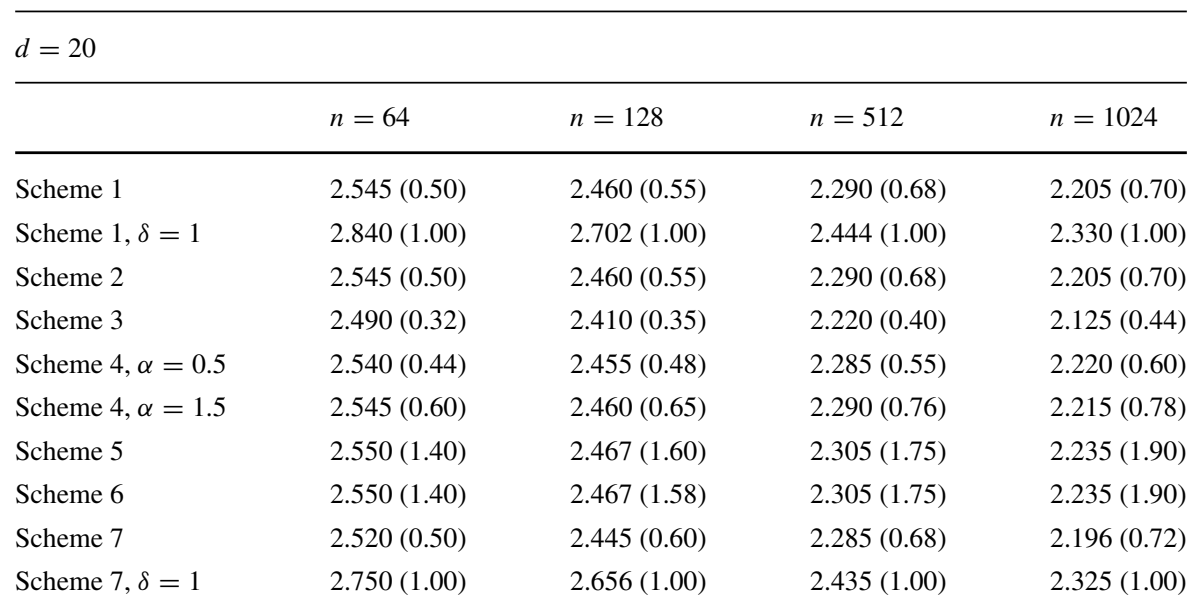


Table 3 Values of $r$ and $\delta$ (in parentheses) to achieve 0.9 coverage for $d=50$

\begin{tabular}{llll}
\hline$d=50$ & & & \\
\hline & $n=128$ & $n=512$ & $n=1024$ \\
\hline Scheme 1 & $4.130(0.38)$ & $4.020(0.45)$ & $3.970(0.46)$ \\
Scheme $1, \delta=1$ & $4.855(1.00)$ & $4.625(1.00)$ & $4.520(1.00)$ \\
Scheme 2 & $4.130(0.38)$ & $4.020(0.45)$ & $3.970(0.46)$ \\
Scheme 3 & $4.110(0.21)$ & $4.000(0.25)$ & $3.950(0.28)$ \\
Scheme $4 \alpha=0.5$ & $4.130(0.30)$ & $4.020(0.36)$ & $3.970(0.40)$ \\
Scheme $4 \alpha=1.5$ & $4.130(0.42)$ & $4.020(0.48)$ & $3.970(0.52)$ \\
Scheme 5 & $4.130(1.50)$ & $4.020(1.75)$ & $3.970(2.00)$ \\
Scheme 6 & $4.130(1.50)$ & $4.020(1.75)$ & $3.970(2.00)$ \\
Scheme 7 & $4.115(0.40)$ & $4.015(0.45)$ & $3.965(0.47)$ \\
Scheme 7, $\delta=1$ & $4.395(1.00)$ & $4.379(1.00)$ & $4.366(1.00)$ \\
\hline
\end{tabular}

In Figs. 12a, 12b, 13a, 13b, 14a, 14b, 15a and 15b we plot $C_{d}\left(\mathbb{Z}_{n}, r\right)$ as a functions of $\delta \in[0,1]$ across a number schemes, $n$ and $d$. For these plots we have used the values of $r$ provided in Tables 1, 2 and 3 such that for Figs. 12a, 12b, 13a and 13b which correspond to Scheme 1 and Scheme 2, the maximum coverage is very close to 0.9 and the optimal $\delta$ is very close to the values presented in Tables 1, 2 and3. For Figs. 12a, 12b, 13a, 13b, 14a, 14b, 15a and 15b the maximum coverage 0.9 is attained with $\delta$ provided in Tables 1, 2 and 3. In Figs. 12a, 12b, 13a, 13b, 14a, 14b, 15a and $15 \mathrm{~b}$ the solid green line, long dashed red line, dashed blue line and dot dashed orange line correspond to $n=64,128,512$, and 1024 respectively. The vertical lines on these plots indicate the value of $\delta$ where the maximum coverage is obtained.

From Tables 1, 2 and 3 and Figs.12a, 12b, 13a, 13b, 14a, 14b, 15a and 15b we arrive at the following conclusions:

- $\quad$ the $\delta$-effect is very important and getting much stronger as $d$ increases;

- coverage of unadjusted low-discrepancy sequences is extremely low;
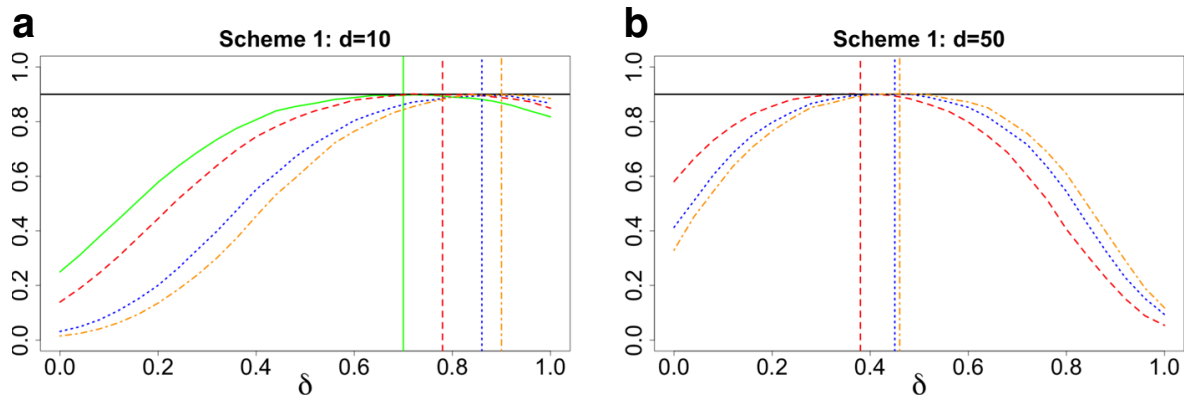

Fig. 12 (a) Scheme 1: $C_{d}\left(\mathbb{Z}_{n}, r\right)$ across $\delta$ for $d=10,(\mathbf{b})$ Scheme 1: $C_{d}\left(\mathbb{Z}_{n}, r\right)$ across $\delta$ for $d=50$ 

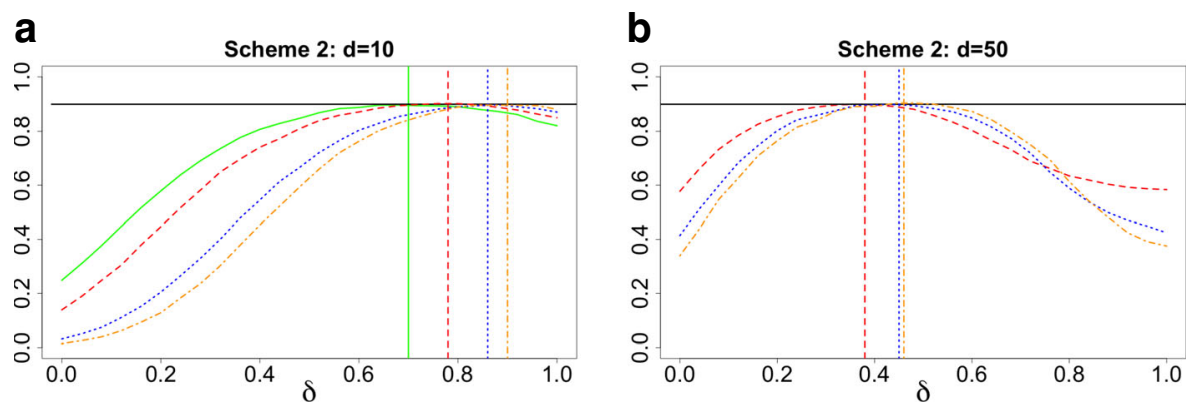

Fig. 13 (a) Scheme 2: $C_{d}\left(\mathbb{Z}_{n}, r\right)$ across $\delta$ for $d=10$, (b) Scheme 2: $C_{d}\left(\mathbb{Z}_{n}, r\right)$ across $\delta$ for $d=50$
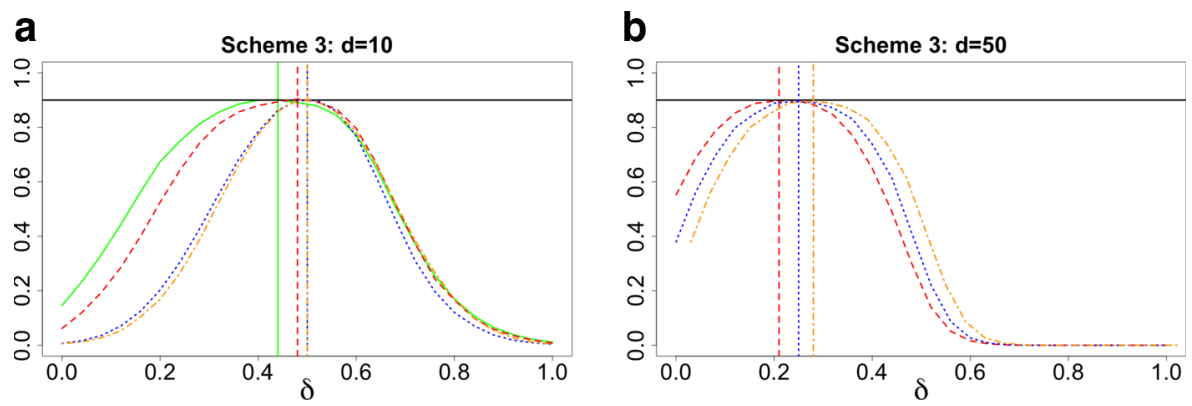

Fig. 14 (a) Scheme 3: $C_{d}\left(\mathbb{Z}_{n}, r\right)$ across $\delta$ for $d=10$, (b) Scheme 3: $C_{d}\left(\mathbb{Z}_{n}, r\right)$ across $\delta$ for $d=50$
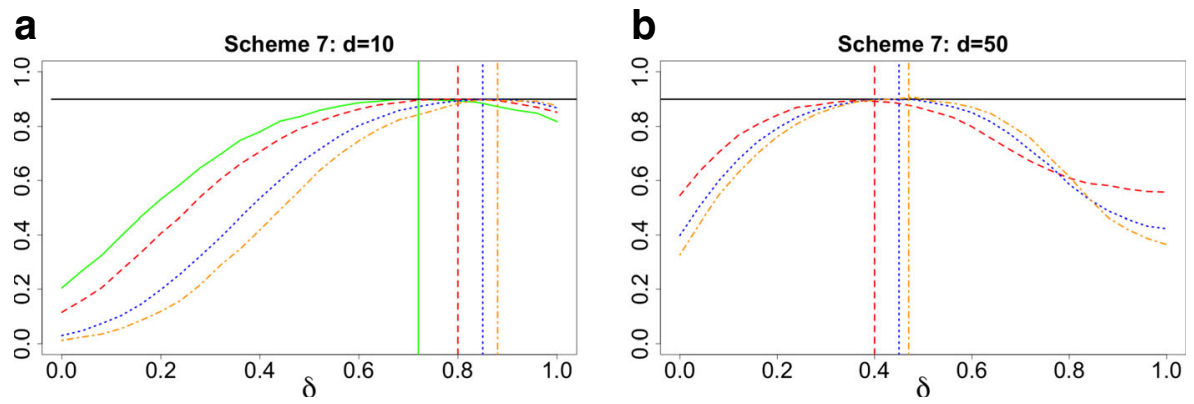

Fig. 15 (a) Scheme 7: $C_{d}\left(\mathbb{Z}_{n}, r\right)$ across $\delta$ for $d=10$, (b) Scheme 7: $C_{d}\left(\mathbb{Z}_{n}, r\right)$ across $\delta$ for $d=50$ 
- properly $\delta$-tuned deterministic Scheme 3, which uses fractional factorial designs of minimum abberation, provides excellent covering;

- randomized Scheme 4 with suitably chosen parameters of the beta-distribution, also provides very high quality coverage (on average);

- for all schemes considered, the coverings with the optimal values of $\delta$ fully comply with the result of Section A1.4 describing the area of volume concentration in the cube $\mathcal{C}_{d}$.

\section{Covering a Cube by Cubes}

\subsection{Volume of Intersection of Two Cubes}

Let us take two cubes: $\mathcal{C}_{d}=[-1,1]^{d}$ and $\mathcal{C}_{d}(Z, r)=\left\{Y \in \mathbb{R}^{d}:\|Y-Z\|_{\infty} \leq r\right\}$, a cube of side length $2 r$ centred at a point $Z=\left(z_{1}, \ldots, z_{d}\right) \in \mathcal{C}_{d}$. Denote the fraction of the cube $\mathcal{C}_{d}$ covered by $\mathcal{C}_{d}(Z, r)$ by

$$
F_{d, Z, r}=\operatorname{vol}\left(\mathcal{C}_{d} \cap \mathcal{C}_{d}(Z, r)\right) / 2^{d} .
$$

Let, like in Section $2.3, U=\left(u_{1}, \ldots, u_{d}\right)$ be a random vector with uniform distribution on $\mathcal{C}_{d}$ so that $u_{1}, \ldots, u_{d}$ are i.i.d.r.v. uniformly distributed on $[-1,1]$. Then

$$
F_{d, Z, r}=\mathbb{P}\left\{\|U-Z\|_{\infty} \leq r\right\}=\mathbb{P}\left\{\max _{1 \leq j \leq d}\left|u_{j}-z_{j}\right| \leq r\right\} .
$$

That is, $F_{d, Z, r}$, as a function of $r$, is the c.d.f. of the r.v. $\|U-Z\|_{\infty}=\max _{1 \leq j \leq d} \mid u_{j}-$ $z_{j} \mid$.

From Lemma 2 of Appendix 2 the c.d.f. of the r.v. $\left|u_{j}-z_{j}\right|$ is

$$
G_{d, z_{j}}(t)=\mathbb{P}\left\{\left|u_{j}-z_{j}\right| \leq t\right\}=\left\{\begin{array}{cl}
0 & \text { for } t \leq 0 \\
t & \text { for } 0<t<1-\left|z_{j}\right| \\
\frac{1}{2}\left(1+t-\left|z_{j}\right|\right) & \text { for } 1-\left|z_{j}\right| \leq t \leq 1+\left|z_{j}\right| \\
1 & 1+\left|z_{j}\right|<t
\end{array}\right.
$$

Since the c.d.f. of a maximum of independent r.v. is the product of marginal c.d.f.'s, we obtain

$$
F_{d, Z, r}=\prod_{j=1}^{d} G_{d, z_{j}}(r) .
$$

Two extreme particular cases of location of $Z$ are:

(i) $Z=0: F_{d, 0, r}=r^{d}, 0 \leq r \leq 1$;

(ii) $\|Z\|=\sqrt{d}$, when $Z$ being a vertex of the cube $\mathcal{C}_{d}: F_{d, V, r}=(r / 2)^{d}, 0 \leq r \leq 2$.

Assume now that we have the cube $\mathcal{C}_{d}(\delta)=[-\delta, \delta]^{d}$ of volume $(2 \delta)^{d}$ and another cube $\mathcal{C}_{d}\left(Z^{\prime}, r^{\prime}\right)=\left\{Y \in \mathbb{R}^{d}:\left\|Y-Z^{\prime}\right\|_{\infty} \leq r^{\prime}\right\}$ with a centre at a point $Z^{\prime}=$ $\left(z_{1}^{\prime}, \ldots, z_{d}^{\prime}\right)$. Denote the fraction of the cube $\mathcal{C}_{d}(\delta)$ covered by $\mathcal{C}_{d}\left(Z^{\prime}, r^{\prime}\right)$ by

$$
F_{d, Z^{\prime}, r^{\prime}}^{(\delta)}=\operatorname{vol}\left(\mathcal{C}_{d}(\delta) \cap \mathcal{C}_{d}\left(Z^{\prime}, r^{\prime}\right)\right) /(2 \delta)^{d} .
$$


Then by changing the coordinates and the radius using (5) we get $F_{d, Z^{\prime}, r^{\prime}}^{(\delta)}=$ $F_{d, Z / \delta, r / \delta}$.

\subsection{Proportion of a Cube Covered by Smaller Cubes with Random Centres}

Let us take the cube $\mathcal{C}_{d}=[-1,1]^{d}$ and $n$ smaller cubes $\mathcal{C}_{d}\left(Z_{j}, r\right)=\left\{Y \in \mathbb{R}^{d}\right.$ : $\left.\left\|Y-Z_{j}\right\|_{\infty} \leq r\right\}$ with centres at points $Z_{j} \in \mathbb{R}^{d}$. Denote the fraction of the cube $\mathcal{C}_{d}$ covered by $\mathcal{C}_{d}\left(\mathbb{Z}_{n}, r\right)=\cup_{j=1}^{n} \mathcal{C}_{d}\left(Z_{j}, r\right)$, the union of these cubes, by

$$
C_{d, \mathbb{Z}_{n}, r}=\operatorname{vol}\left(\mathcal{C}_{d} \cap \mathcal{C}_{d}\left(\mathbb{Z}_{n}, r\right)\right) / 2^{d}
$$

Our aim is to obtain a closed form expression for this quantity for arbitrary $d, r$ and $n$ in the case when $Z_{1}, \ldots, Z_{n}$ are i.i.d. random vectors uniformly distributed in the cube $\mathcal{C}_{d}(\delta)=[-\delta, \delta]^{d}$ with $0<\delta \leq 1$. $\mathbb{R}^{d}$,

Similarly to the combination of (19) with (21), for a given $U=\left(u_{1}, \ldots, u_{d}\right) \in$

$$
\mathbb{P}\left\{U \in \mathcal{C}_{d}\left(\mathbb{Z}_{n}, r\right)\right\}=1-\left(1-F_{d, U / \delta, r / \delta}\right)^{n} .
$$

Similarly to (20),

$$
C_{d, \mathbb{Z}_{n}, r}=\mathbb{E}_{U} \mathbb{P}\left\{U \in \mathcal{C}_{d}\left(\mathbb{Z}_{n}, r\right)\right\}=1-\mathbb{E}_{U}\left(1-F_{d, U / \delta, r / \delta}\right)^{n} .
$$

For an integer $k$, set

$$
I_{k}=\frac{1}{2} \int_{-1}^{1}\left[G_{d, u / \delta}(r / \delta)\right]^{k} d u .
$$

Then, using the binomial theorem, we have

$$
C_{d, \mathbb{Z}_{n}, r}=1-\sum_{k=0}^{n}(-1)^{k}\left(\begin{array}{l}
n \\
k
\end{array}\right) I_{k}^{d} .
$$

It is possible to evaluate (28) explicitly. For $k=0$ and for $r \geq \delta+1$, we clearly have $I_{k}=1$. For $k \geq 1$ and $0 \leq r \leq \delta+1$, the integral $I_{k}$ takes different forms depending on the values of $r$ and $\delta$ :

$$
I_{k}=\left\{\begin{array}{cl}
(\delta-r)\left(\frac{r}{\delta}\right)^{k}-\frac{2 \delta}{(k+1)}\left\{\left(\frac{\delta+r-1}{2 \delta}\right)^{k+1}-\left(\frac{r}{\delta}\right)^{k+1}\right\} & \text { for } r \leq \delta \\
(r-\delta)-\frac{2 \delta}{(k+1)}\left\{\left(\frac{\delta+r-1}{2 \delta}\right)^{k+1}-1\right\} & \text { for } 0 \leq r-\delta \leq 1, r+\delta \geq 1 \\
(r-\delta)+2 \delta /(k+1) & \text { for } 0 \leq r-\delta \leq 1, r+\delta \leq 1 .
\end{array}\right.
$$

In Figs. 16a and 16b, we depict values of $C_{d, \mathbb{Z}_{n}, r}$ (computed using (29)) as a function of $\delta$ for a number of choices of $r$. As in Section 3.5, we note that the $\delta$-effect holds for the problem of coverage of the cube by smaller cubes. 

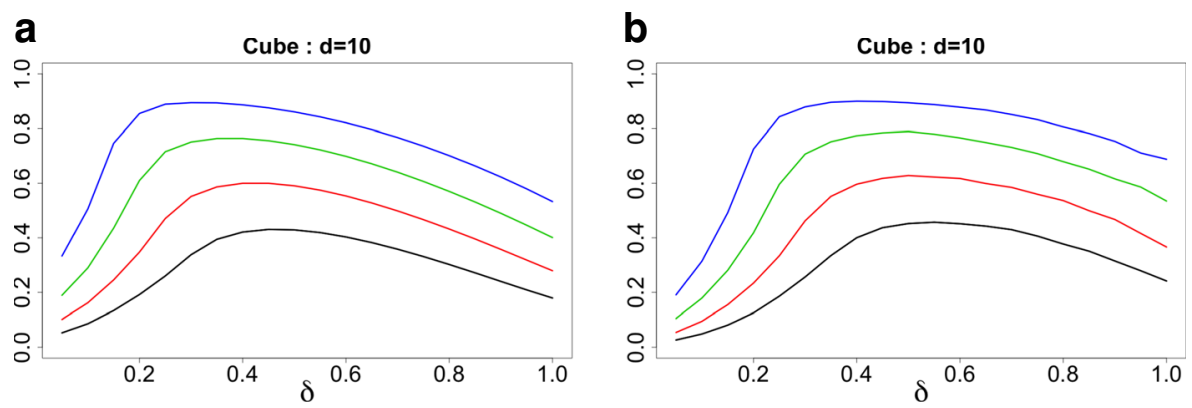

Fig. 16 (a) $n=50, r \in[0.7,0.85]$ increasing by 0.05, (b) $n=128, r \in[0.6,0.8]$ increasing by 0.05

\section{Quantization}

In this section, we briefly consider the following problem of quantization also known as the problem of facility location. Let $X=\left(x_{1}, \ldots, x_{d}\right)$ be uniform on $\mathcal{C}_{d}=[-1,1]^{d}$ and $\mathbb{Z}_{n}=\left\{Z_{1}, \ldots, Z_{n}\right\}$ be an $n$-point design. The mean square quantization error is $\theta_{n}=\theta\left(\mathbb{Z}_{n}\right)=\mathbb{E}_{X} \min _{i=1, \ldots, n}\left\|X-Z_{i}\right\|^{2}$. In the case where $Z_{1}, \ldots, Z_{n}$ are i.i.d. uniform on $\mathcal{C}_{d}(\delta)$, we will derive a simple approximation for the expected value of $\theta\left(\mathbb{Z}_{n}\right)$ in order to demonstrate the $\delta$-effect. We shall also notice a strong correlation in design efficiency used for quantization and for $(1-\gamma)$-covering as studied in Section 3.

The two characteristics, $C_{d}\left(\mathbb{Z}_{n}, r\right)$ and $\theta\left(\mathbb{Z}_{n}\right)$, are related as follows. $\mathbb{E}_{\mathbb{Z}_{n}} C_{d}\left(\mathbb{Z}_{n}, r\right)$, as a function of $r \geq 0$, is the c.d.f. of the r.v. $\mathbb{E}_{\mathbb{Z}_{n}} \min _{i=1, \ldots, n}\left\|X-Z_{i}\right\|$, where $X$ is uniform on $[-1,1]^{d}$, while $\mathbb{E}_{\mathbb{Z}_{n}} \theta\left(\mathbb{Z}_{n}\right)$ is the second moment of the distribution with this c.d.f.:

$$
\mathbb{E}_{\mathbb{Z}_{n}} \theta\left(\mathbb{Z}_{n}\right)=\mathbb{E}_{\mathbb{Z}_{n}} \mathbb{E}_{X} \min _{i=1, \ldots, n}\left\|X-Z_{i}\right\|^{2}=\mathbb{E}_{X} \mathbb{E}_{\mathbb{Z}_{n}} \min _{i=1, \ldots, n}\left\|X-Z_{i}\right\|^{2}=\int_{r \geq 0} r^{2} d \mathbb{E}_{\mathbb{Z}_{n}} C_{d}\left(\mathbb{Z}_{n}, r\right) .
$$

Using approximation (26) we obtain

$$
\begin{aligned}
\frac{d}{d r}\left(\mathbb{E}_{\mathbb{Z}_{n}} C_{d}\left(\mathbb{Z}_{n}, r\right)\right) \cong f_{\delta}(r) & :=\frac{n \cdot r}{\delta} \int_{-\infty}^{\infty} \frac{\varphi(s) \varphi\left(c_{s}\right) \psi_{2}(s)}{\sqrt{s^{\prime}+k}} \times \\
& \times\left[\sqrt{3}+\left(1+\frac{4}{d}\right) \frac{\left(s^{\prime}+\frac{d \delta^{2}}{63}\right)}{5\left(s^{\prime}+k\right)^{3 / 2}}\left\{\delta\left(c_{s}^{3}-c_{s}\right)-\frac{\sqrt{3}\left(r^{2}-\frac{d \delta^{2}}{3}-s^{\prime}\right)}{\sqrt{s^{\prime}+k}}\right\}\right] d s
\end{aligned}
$$

with

$$
\psi_{2}(s)=\exp \left\{-n\left(\Phi\left(c_{s}\right)+\left(1+\frac{4}{d}\right) \frac{\delta\left[s^{\prime}+\frac{d \delta^{2}}{63}\right]}{5 \sqrt{3}\left[s^{\prime}+k\right]^{3 / 2}}\left(1-c_{s}^{2}\right) \phi\left(c_{s}\right)\right)\right\}
$$

and

$$
c_{s}=\frac{\sqrt{3}\left(r^{2}-s^{\prime}-\frac{d \delta^{2}}{3}\right)}{2 \delta \sqrt{s^{\prime}+k}}, s^{\prime}=s \sqrt{\frac{4 d}{45}}+d / 3, k=\frac{d \delta^{2}}{15} \text {. }
$$



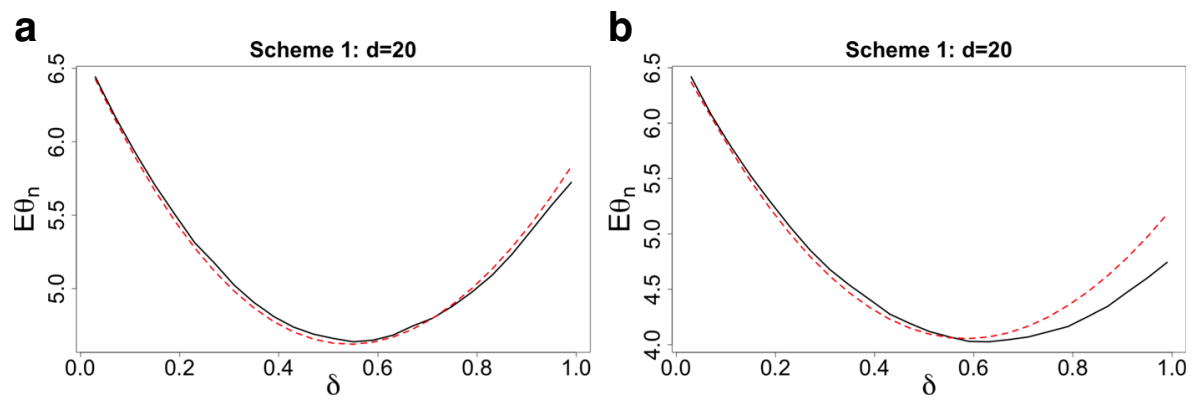

Fig. 17 (a) $\mathbb{E} \theta_{n}$ and approximation (31); $n=128$, (b) $\mathbb{E} \theta_{n}$ and approximation (31); $n=512$

Therefore using relation (30) the approximation for $\mathbb{E}_{\mathbb{Z}_{n}} \theta\left(\mathbb{Z}_{n}\right)$ for Scheme 1 is:

$$
\mathbb{E} \theta_{n}=\mathbb{E}_{\mathbb{Z}_{n}} \theta\left(\mathbb{Z}_{n}\right) \cong \int_{r \geq 0} r^{2} f_{\delta}(r) d r
$$

In Figs. 17a, 17b, 18a and 18b, we asses the accuracy of the approximation (31). In these figures, the solid black line corresponds to $\mathbb{E} \theta_{n}$ obtained via Monte Carlo methods and the dashed red line depicts the approximation. We see that the accuracy of approximation (31) is very high for small $n$ and large $d$. However, as Fig. 17b shows, if $d$ is not large enough but $n$ is large then the errors accumulate and the resulting approximation may not be accurate enough.

As follows from results of [8, Ch.6], for efficient covering schemes the order of convergence of the covering radius to 0 as $n \rightarrow \infty$ is $n^{-1 / d}$. Therefore, for the mean squared distance (which is the quantization error) we should expect the order $n^{-2 / d}$ as $n \rightarrow \infty$. Therefore, for sake of comparison of quantization errors $\theta_{n}$ across $n$ we renormalize this error from $\mathbb{E} \theta_{n}$ to $n^{2 / d} \mathbb{E} \theta_{n}$.

In Tables 4, 5 and 6, we present the minimum value of $n^{2 / d} \mathbb{E} \theta_{n}$ for a selection of the schemes among those considered in Section 3. In these tables, the value within the parentheses corresponds to the value of $\delta$ where the minimum of $n^{2 / d} \mathbb{E} \theta_{n}$ was obtained. For Scheme 3, typical behaviour of $\mathbb{E} \theta_{n}$ across $\delta$ for a number and $n$ and $d$ is presented in Figs. 19a, 19b, 20a and 20b.
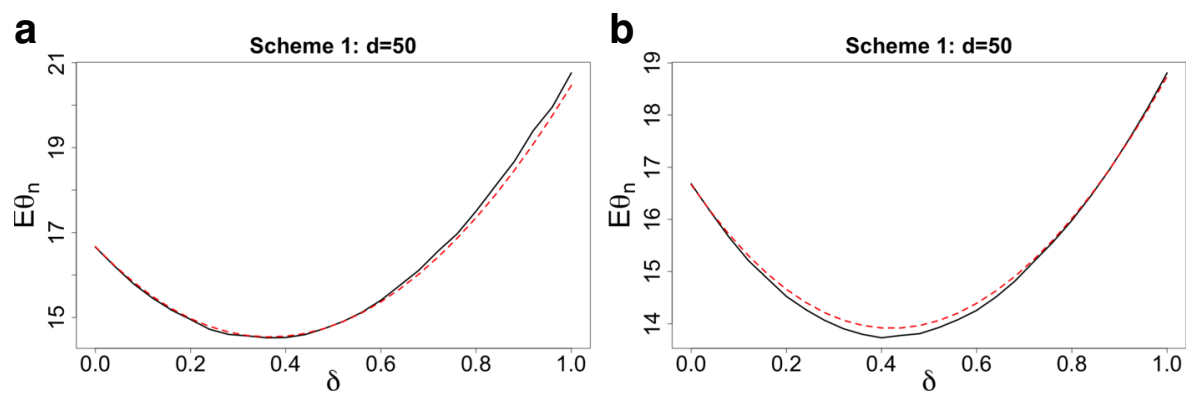

Fig. 18 (a) $\mathbb{E} \theta_{n}$ and approximation (31); $n=128$, (b) $\mathbb{E} \theta_{n}$ and approximation (31); $n=512$ 
Table 4 Minimum value of $n^{2 / d} \mathbb{E} \theta_{n}$ and $\delta$ (in parentheses) across schemes and $n$ for $d=10$

\begin{tabular}{lllll}
\hline$d=10$ & & & & \\
\hline & $n=64$ & $n=128$ & $n=512$ & $n=1024$ \\
\hline Scheme 1 & $4.153(0.68)$ & $4.105(0.72)$ & $3.992(0.80)$ & $3.925(0.84)$ \\
Scheme 3 & $3.663(0.40)$ & $3.548(0.44)$ & $3.221(0.48)$ & $3.348(0.52)$ \\
Scheme 4, $\alpha=0.5$ & $4.072(0.56)$ & $4.013(0.60)$ & $3.839(0.68)$ & $3.770(0.69)$ \\
Scheme 7 & $3.998(0.68)$ & $3.973(0.76)$ & $3.936(0.80)$ & $3.834(0.82)$ \\
Scheme 7, $\delta=1$ & $4.569(1.00)$ & $4.425(1.00)$ & $4.239(1.00)$ & $4.094(1.00)$ \\
\hline
\end{tabular}

Table 5 Minimum value of $n^{2 / d} \mathbb{E} \theta_{n}$ and $\delta$ (in parentheses) across schemes and $n$ for $d=20$

\begin{tabular}{lllll}
\hline$d=20$ & & & & \\
\hline & $n=64$ & $n=128$ & $n=512$ & $n=1024$ \\
\hline Scheme 1 & $7.552(0.52)$ & $7.563(0.56)$ & $7.528(0.64)$ & $7.484(0.68)$ \\
Scheme 3 & $7.298(0.32)$ & $7.270(0.33)$ & $7.133(0.36)$ & $7.016(0.40)$ \\
Scheme 4, $\alpha=0.5$ & $7.541(0.40)$ & $7.515(0.44)$ & $7.457(0.52)$ & $7.421(0.54)$ \\
Scheme 7 & $7.445(0.48)$ & $7.464(0.56)$ & $7.487(0.64)$ & $7.453(0.66)$ \\
Scheme 7, $\delta=1$ & $9.089(1.00)$ & $9.133(1.00)$ & $8.87(1.00)$ & $8.681(1.00)$ \\
\hline
\end{tabular}

Table 6 Minimum value of $n^{2 / d} \mathbb{E} \theta_{n}$ and $\delta$ (in parentheses) across schemes and $n$ for $d=50$.

\begin{tabular}{llll}
$d=50$ & & & \\
\hline & $n=128$ & $n=512$ & $n=1024$ \\
\hline Scheme 1 & $17.608(0.36)$ & $17.634(0.40)$ & $17.643(0.44)$ \\
Scheme 3 & $17.483(0.20)$ & $17.511(0.24)$ & $17.554(0.27)$ \\
Scheme 4, $\alpha=0.5$ & $17.590(0.28)$ & $17.670(0.36)$ & $17.620(0.38)$ \\
Scheme 7, $\delta=1$ & $20.196(1.00)$ & $21.231(1.00)$ & $21.711(1.00)$ \\
\hline
\end{tabular}
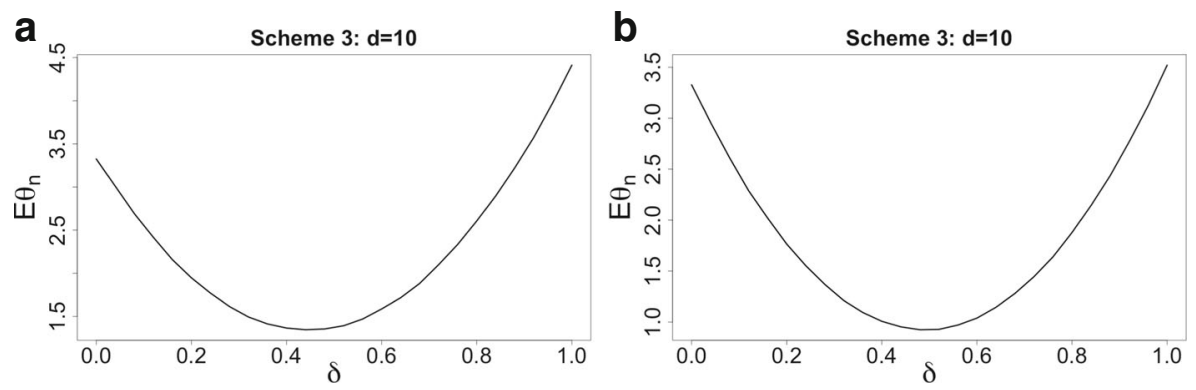

Fig. 19 (a) $\mathbb{E} \theta_{n}$ with $n=128$, (a) $\mathbb{E} \theta_{n}$ with $n=512$ 
a

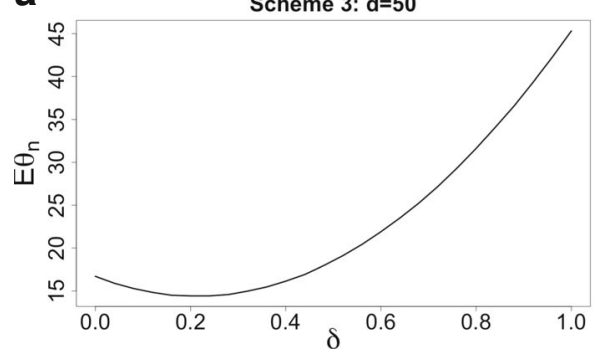

b

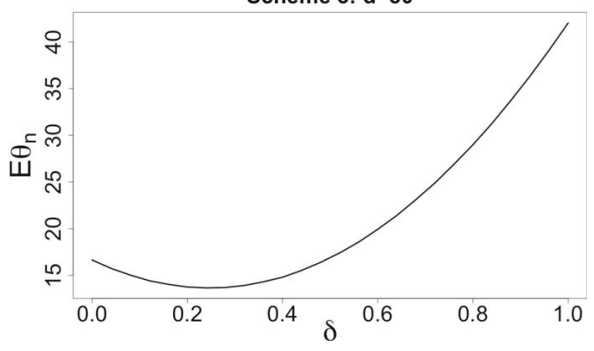

Fig. 20 (a) $\mathbb{E} \theta_{n}$ with $n=128$, (b) $\mathbb{E} \theta_{n}$ with $n=512$

We make the following two main conclusions from analysing results of this numerical study:

(a) the presence of a strong $\delta$-effect, very similar to the effect observed in Section 3 , and

(b) for a given design $\mathbb{Z}_{n}$, there is a very strong correlation between the covering probability as studied in Section 3 and the normalized quantization error $n^{2 / d} \mathbb{E} \theta\left(\mathbb{Z}_{n}\right)$.

By comparing the values of $\delta$ in Tables 4, 5 and 6 with those in Tables 1, 2 and 3 , we see a strong similarity between efficient quantization schemes and efficient covering schemes.

Funding Information Jack Noonan was financially supported by the EPSRC grant number EP/N509449/1.

\section{Compliance with Ethical Standards}

Conflict of Interest The authors declare that they have no conflict of interest.

Open Access This article is licensed under a Creative Commons Attribution 4.0 International License, which permits use, sharing, adaptation, distribution and reproduction in any medium or format, as long as you give appropriate credit to the original author(s) and the source, provide a link to the Creative Commons licence, and indicate if changes were made. The images or other third party material in this article are included in the article's Creative Commons licence, unless indicated otherwise in a credit line to the material. If material is not included in the article's Creative Commons licence and your intended use is not permitted by statutory regulation or exceeds the permitted use, you will need to obtain permission directly from the copyright holder. To view a copy of this licence, visit http://creativecommonshorg/licenses/by/4. $0 /$.

\section{Appendix 1. Several Facts about $d$-Dimensional Balls and Cubes}

In this appendix, we briefly mention several facts, used in the main part of the paper, related to high-dimensional cubes and balls. Many of these facts are somewhat 
Table 7 Radius of the ball of unit volume for different dimensions

\begin{tabular}{llllllllll}
\hline$d$ & 1 & 2 & 3 & 4 & 5 & 6 & 7 & 8 & 9 \\
$r_{d}$ & 0.5 & 0.564 & 0.62 & 0.671 & 0.717 & 0.761 & 0.8 & 0.839 & 0.876 \\
$d$ & 10 & 20 & 30 & 40 & 50 & 100 & 200 & 500 & 1000 \\
$r_{d}$ & 0.911 & 1.201 & 1.43 & 1.626 & 1.8 & 2.49 & 3.477 & 5.45 & 7.682 \\
\hline
\end{tabular}

counter-intuitive and often lead to creation of wrong heuristics in multivariate optimization and misunderstanding of the behaviour of even simple algorithms in highdimensional spaces. For more details concerning the material of Sections A1.1-A1.4, see [1].

\section{A1.1. Volume of the Ball}

The volume of the ball $\mathcal{B}_{d}(r)=\left\{x \in \mathbb{R}^{d}:\|x\| \leq r\right\}$ can be computed by the formula

$$
\operatorname{vol}\left(\mathcal{B}_{d}(r)\right)=r^{d} V_{d}, \text { where } V_{d}=\operatorname{vol}\left(\mathcal{B}_{d}(1)\right)=\frac{\pi^{d / 2}}{\Gamma(d / 2+1)}
$$

The volumes $V_{d}$ decrease very fast as $d$ grows. For example, $V_{100} \simeq 2.368 \cdot 10^{-40}$. As $d \rightarrow \infty$,

$$
V_{d}^{1 / d} \simeq \sqrt{2 \pi e} \frac{1}{\sqrt{d}}+O\left(\frac{\log d}{d^{3 / 2}}\right)
$$

\section{A1.2. Radius of the Ball of Unit Volume}

Define $r_{d}$ by $\operatorname{vol}\left(\mathcal{B}_{d}\left(r_{d}\right)\right)=1$. Table 7 gives approximate values of $r_{d}$.

From (33), for large $d$ we have

$$
r_{d}=\frac{\sqrt{d}}{\sqrt{2 \pi e}}+O\left(\frac{1}{\sqrt{d}}\right)
$$

where $1 / \sqrt{2 \pi e} \simeq 0.242$. This is only about twice smaller than $\sqrt{d} / 2$, the length of the half-diagonal of the $d$-dimensional unit cube $[0,1]^{d}$.

For $r_{d, 2 \delta}$ defined by $\operatorname{vol}\left(\mathcal{B}_{d}\left(r_{d, 2 \delta}\right)\right)=\operatorname{vol}\left(\mathcal{C}_{d}(\delta)\right)=(2 \delta)^{d}$, we have $r_{d, 2 \delta}=2 \delta r_{d}$.

\section{A1.3. Almost All the Volume is Near the Boundary}

First, consider the cube $\mathcal{C}_{d}(\delta)=[-\delta, \delta]^{d}$, with $0<\delta<1$, as interior to the cube $\mathcal{C}_{d}=[-1,1]^{d}$. For the ratio of the volumes of these two cubes, we have $\operatorname{vol}\left(\mathcal{C}_{d}(\delta)\right) / \operatorname{vol}\left(\mathcal{C}_{d}\right)=\delta^{d}$ which tends to 0 (as $d \rightarrow \infty$ ) exponentially fast for any $\delta \in(0,1)$. 
If, as $d \rightarrow \infty, \delta$ changes getting closer to 1 but $1-\delta$ tends to 0 slower than $1 / d$, then the ratio of the two volumes still tends to 0 . In particular, if $1-\delta=c / d^{1-\delta}$ with $0<\delta<1$ then

$$
\frac{\operatorname{vol}\left(\mathcal{C}_{d}(\delta)\right)}{\operatorname{vol}\left(\mathcal{C}_{d}\right)}=\delta^{d} \simeq \exp \left\{-c d^{1-\delta}\right\} \rightarrow 0, \quad d \rightarrow \infty .
$$

Consider now the balls $\mathcal{B}_{d}(1)$ and $\mathcal{B}_{d}(1-\epsilon)$. The difference $\mathcal{B}_{d}(1) \backslash \mathcal{B}_{d}(1-\epsilon)$ is called the annulus. Using (32) we can compute the ratio of volume of this annulus to the volume of the unit ball:

$$
\frac{\operatorname{vol}\left[\mathcal{B}_{d}(1) \backslash \mathcal{B}_{d}(1-\epsilon)\right]}{\operatorname{vol}\left(\mathcal{B}_{d}(1)\right)}=1-\varepsilon^{d} .
$$

This ratio tends to 1 exponentially fast as $d \rightarrow \infty$. The ratio of volume of the ball $\mathcal{B}_{d}(1-\epsilon)$ to the volume of the unit ball $\mathcal{B}_{d}(1)$ is, similarly to the case of the cubes above, $(1-\varepsilon)^{d}$. This result extends to any measurable set $A \subset \mathbb{R}^{d}$. Indeed, define the set $A_{1-\varepsilon}=\{(1-\varepsilon) x: x \in A\}$. Then, by splitting $A$ and $A_{1-\varepsilon}$ into infinitesimal cubes and adding up their volumes, we find $\operatorname{vol}\left(A_{1-\varepsilon}\right)=(1-\varepsilon)^{d} \operatorname{vol}(A)$.

\section{A1.4. The Area of Volume Concentration in a Cube}

Let $X=\left(x_{1}, \ldots, x_{d}\right)$ be uniformly distributed on $\mathcal{C}_{d}=[-1,1]^{d}$. Then $x_{1}^{2}, \ldots, x_{d}^{2}$ are independent r.v. on $[0,1]$. The Hoeffding's inequality gives

$$
\mathbb{P}\left\{\left|\frac{1}{d}\left(x_{1}^{2}+\ldots+x_{d}^{2}\right)-\frac{1}{d} \mathbb{E}\left(x_{1}^{2}+\ldots+x_{d}^{2}\right)\right| \geq \epsilon\right\} \leq 2 e^{-2 d \epsilon^{2}} .
$$

Since $\mathbb{E} x_{i}^{2}=\frac{1}{3}$, we obtain

$$
\mathbb{P}\left\{\left|\|X\|^{2}-\frac{d}{3}\right| \geq \epsilon d\right\} \leq 2 e^{-2 d \epsilon^{2}} .
$$

Therefore, the main volume in the cube $\mathcal{C}_{d}$ is concentrated in the annulus around the sphere with radius $\sqrt{d / 3}$.

\section{A1.5. Squared Norm of a Random Point in a Cube}

Let $Z=\left(z_{1}, \ldots, z_{d}\right)$ be a random vectors on $\mathcal{C}_{d}(\delta)=[-\delta, \delta]^{d}$ consisting of i.i.d. random components $z_{i}$ having a distribution with density $p(t), t \in[-\delta, \delta], \delta>0$.

Set $\eta=\sum_{j=1}^{d} z_{j}^{2}$. We have $\mathbb{E} \eta=d \mu_{2}$ and $\operatorname{var}(\eta)=d \operatorname{var}\left(z_{1}^{2}\right)=d\left(\mu_{4}-\mu_{2}^{2}\right)$, where $\mu_{j}$ be the moments of the distribution with density $p(t)$.

For example, when $z_{i}$ have $\operatorname{Beta}(\alpha, \alpha)$ distribution with density

$$
p_{\alpha, \delta}(t)=\frac{(2 \delta)^{1-2 \alpha}}{\operatorname{Beta}(\alpha, \alpha)}\left[\delta^{2}-t^{2}\right]^{\alpha-1}, \quad-\delta<t<\delta, \alpha>0,
$$

where $\operatorname{Beta}(\cdot, \cdot)$ is the beta-function, then

$$
\mu_{2}=\frac{\delta^{2}}{2 \alpha+1}, \mu_{4}=\frac{3 \delta^{4}}{(2 \alpha+1)(2 \alpha+3)}
$$


and therefore

$$
\mathbb{E} \eta=\frac{d \delta^{2}}{2 \alpha+1}, \quad \operatorname{var}(\eta)=\frac{4 d \delta^{4} \alpha}{(2 \alpha+1)^{2}(2 \alpha+3)} .
$$

If $\alpha=1$, when $Z$ is uniform in the cube $\mathcal{C}_{d}(\delta)$, then

$$
\mathbb{E} \eta=\frac{1}{3} d \delta^{2}, \quad \operatorname{var}(\theta)=\frac{4}{45} d \delta^{4} .
$$

\section{A1.6. Distance Between Two Random Points in a Cube}

Assume $Z=\left(z_{1}, \ldots, z_{d}\right)$ and $Z^{\prime}=\left(z_{1}^{\prime}, \ldots, z_{d}^{\prime}\right)$ are independent random vectors on $\mathcal{C}_{d}(\delta)=[-\delta, \delta]^{d}$ consisting of i.i.d. random components $z_{i}$ and $z_{i}^{\prime}$ which have some distribution with density $p(t), t \in[-\delta, \delta], \delta>0$. Let $\mu_{j}$ be the moments of the distribution with density $p(t)$. Assume that the density $p(t)$ is symmetric around 0 and hence all odd moments are zero: $\mu_{2 k+1}=0$ for $k=1,2, \ldots$

The distribution of the squared distances

$$
\theta=\left\|Z-Z^{\prime}\right\|^{2}=\sum_{i=1}^{d}\left(z_{i}-z_{i}^{\prime}\right)^{2}
$$

has the mean and variance that can be easily computed as follows:

$$
\begin{aligned}
\mathbb{E} \theta & =d \mathbb{E}\left(z_{1}-z_{1}^{\prime}\right)^{2}=2 d \mu_{2}, \\
\operatorname{var}(\theta)=d \operatorname{var}\left(z_{1}-z_{1}^{\prime}\right)^{2} & =d\left[\left[\mathbb{E}\left(z_{1}-z_{1}^{\prime}\right)^{4}-\left[\mathbb{E}\left(z_{1}-z_{1}^{\prime}\right)^{2}\right)\right]^{2}\right]=2 d\left[\mu_{4}+\mu_{2}^{2}\right]
\end{aligned}
$$

For example, when $z_{i}$ and $z_{i}^{\prime}$ have $\operatorname{Beta}(\alpha, \alpha)$ distribution with density (34) and hence moments (35), we obtain

$$
\mathbb{E} \theta=\frac{2 d \delta^{2}}{2 \alpha+1}, \quad \operatorname{var}(\theta)=\frac{4 d \delta^{4}(4 \alpha+3)}{(2 \alpha+1)^{2}(2 \alpha+3)} .
$$

If $\alpha=1$ (that is, when $Z$ and $Z^{\prime}$ are uniform in the cube $\mathcal{C}_{d}(\delta)$ ), then

$$
\mathbb{E} \theta=\frac{2}{3} d \delta^{2}, \quad \operatorname{var}(\theta)=\frac{28}{45} d \delta^{4}
$$

\section{A1.7. Volume of the Intersection of Two Balls of the Same Radius}

Let $\mathcal{B}_{d}\left(Z_{j}, r\right)$ and $\mathcal{B}_{d}\left(Z_{i}, r\right)$ be two balls in $\mathbb{R}^{d}$ with same radius and different centres $Z$ and $Z^{\prime}$. To compute the volume of the intersection $\mathcal{B}_{d}(Z, r) \cap \mathcal{B}_{d}\left(Z^{\prime}, r\right)$, we will use the formula for the volume of the $d$-dimensional cap (cut in the direction of $Z^{\prime}$ ) of height $h$ from a $d$-dimensional ball $\mathcal{B}_{d}(Z, r)$ :

$$
K_{d, r, h}=\frac{1}{2} r^{d} V_{d} I_{1-h^{2} / r^{2}}\left(\frac{d-1}{2}, \frac{1}{2}\right)-\frac{h}{d}\left(r^{2}-h^{2}\right)^{(d-1) / 2} V_{d-1},
$$

where $V_{d}$ is defined in (32), $\Gamma(\cdot)$ is the gamma-function and

$$
I_{t}(\alpha, \beta)=\int_{0}^{t} u^{\alpha-1}(1-u)^{\beta-1} d u / \int_{0}^{1} u^{\alpha-1}(1-u)^{\beta-1} d u
$$


is the normalized incomplete beta-function. In the rhs of (40), the first term is the volume of the related $d$-dimensional hyper-sector (this expression is derived in [7]) and the second term is the volume of the cone with height $h$ and base $\mathcal{B}_{d-1}((Z+$ $\left.\left.Z^{\prime}\right) / 2, r^{\prime}\right)$, where $r^{\prime}=\sqrt{r^{2}-h^{2}}$.

The volume of the intersection of the balls $\mathcal{B}_{d}(Z, r)$ and $\mathcal{B}_{d}\left(Z^{\prime}, r\right)$ is therefore

$$
\operatorname{vol}\left(\mathcal{B}_{d}(Z, r) \cap \mathcal{B}_{d}\left(Z^{\prime}, r\right)\right)=2 K_{d, r, h}
$$

where $h=\frac{1}{2}\left\|Z-Z^{\prime}\right\|$ and $K_{d, r, h}$ is defined in (40).

\section{A1.8. A Direct Computation of $C_{d, z, r}$}

For computing values of $C_{d, Z, r}$, we can employ the following direct approach based on the use of characteristic functions (c.f.).

(a) Compute the c.f. $\psi_{z}(s)=\int e^{i t s} \varphi_{z}(t) d t$ for $z=z_{j}(j=1, \ldots, d)$, with the density $\varphi_{z}(t)$ defined either by (7) or (9).

(b) As $u_{j}$ are independent, the c.f. of $\|U-Z\|^{2}$ is the product $\psi_{Z}(s)=$ $\prod_{j=1}^{d} \psi_{z_{j}}(s)$.

(c) The density of $\|U-Z\|^{2}$ is found using the inversion formula

$$
p_{d, Z}(x)=\frac{1}{2 \pi} \int_{-\infty}^{\infty} e^{-i s x} \psi_{Z}(s) d s, \quad x \geq 0 .
$$

For computing the c.f. $\psi_{z}(s)=\int e^{i t s} \varphi_{z}(t) d t$ we can use the formula

$$
\int_{a}^{b} \frac{e^{x t}}{\sqrt{t}} d t=2 \int_{\sqrt{a}}^{\sqrt{b}} e^{x u^{2}} d u=\sqrt{\frac{\pi}{x}}(\operatorname{erfi}(\sqrt{b x})-\operatorname{erfi}(\sqrt{a x}))
$$

for any $0 \leq a<b<\infty$ and any complex $x \neq 0$. Here erfi $(x)$ is the imaginary error function

$$
\operatorname{erfi}(x)=\frac{2}{\sqrt{\pi}} \int_{0}^{x} e^{t^{2}} d t=\frac{2}{\sqrt{\pi}} \sum_{j=0}^{\infty} \frac{x^{2 j+1}}{j !(2 j+1)} ;
$$

the series in the right-hand side of this formula converges for all complex $x$.

This approach allows very accurate computation of $C_{d, Z, r}$ but it is very computationally intensive and can only be performed for given $Z$.

\section{Appendix 2. Important Auxiliary Results}

Lemma 1 Let $\delta>0, x \in \mathbb{R}$ and $\eta_{x, \delta}$ be a r.v. $\eta_{x, \delta}=(\xi-x)^{2}$, where r.v. $\xi$ has uniform distribution on $[-\delta, \delta]$. Then the c.d.f. of the r.v. $\eta_{x, \delta}$ is

$$
F_{x, \delta}(t)=\mathbb{P}\left\{\eta_{x, \delta} \leq t\right\}= \begin{cases}0 & \text { for } t \leq 0 \\ \frac{\sqrt{t}}{\delta} \cdot 1_{[|x| \leq \delta]} & \text { for } 0<t<(\delta-|x|)^{2} \\ \frac{\delta-|x|+\sqrt{t}}{2 \delta} & \text { for }(\delta-|x|)^{2} \leq t \leq(\delta+|x|)^{2} \\ 1 & (\delta+|x|)^{2}<t,\end{cases}
$$


where

$$
1_{[|x| \leq \delta]}=\left\{\begin{array}{l}
1 \text { if }|x| \leq \delta \\
0 \text { if }|x|>\delta
\end{array}\right.
$$

The corresponding density of $\eta_{x, \delta}$ is

$$
\varphi_{x, \delta}(t)= \begin{cases}1 /(2 \delta \sqrt{t}) \cdot 1_{[|x| \leq \delta]} & \text { for } 0<t<(\delta-|x|)^{2} \\ 1 /(4 \delta \sqrt{t}) & \text { for }(\delta-|x|)^{2}<t \leq(\delta+|x|)^{2} \\ 0 & \text { otherwise. }\end{cases}
$$

The first four central moments of the r.v. $\eta_{x, \delta}$ are:

$$
\begin{gathered}
\mu_{x, \delta}^{(1)}=E \eta_{x, \delta}=x^{2}+\frac{\delta^{2}}{3}, \quad \mu_{x, \delta}^{(2)}=\operatorname{var}\left(\eta_{x, \delta}\right)=\frac{4 \delta^{2}}{3}\left(x^{2}+\frac{\delta^{2}}{15}\right), \\
\mu_{x, \delta}^{(3)}=E\left[\eta_{x, \delta}-E \eta_{x, \delta}\right]^{3}=\frac{16 \delta^{4}}{15}\left(x^{2}+\frac{\delta^{2}}{63}\right), \\
\mu_{x, \delta}^{(4)}=E\left[\eta_{x, \delta}-E \eta_{x, \delta}\right]^{4}=3 \mu_{x, \delta}^{(1)} \mu_{x, \delta}^{(3)} .
\end{gathered}
$$

Proof Clearly, if $t \leq 0$ then $F_{x, \delta}(t)=0$ and so we only consider the case $t>0$. In view of symmetry, for all $x \in \mathbb{R}, \delta>0$ and $t \geq 0$, we have $F_{x, \delta}(t)=F_{-x, \delta}(t)$ and therefore we only need to consider $x \geq 0$. Also, $\eta_{x, \delta} \leq(|x|+\delta)^{2}$ with probability 1 implying $F_{x, \delta}(t)=1$ for all $t \geq(|x|+\delta)^{2}$.

Assume $0 \leq x \leq \delta$. We then have for all $t \geq 0$ :

$$
\begin{aligned}
F_{x, \delta}(t) & =\mathbb{P}\left\{(\xi-x)^{2} \leq t\right\}=\mathbb{P}\left\{(\xi-x)^{2} \leq t, \xi \leq x\right\}+\mathbb{P}\left\{(\xi-x)^{2} \leq t, \xi>x\right\} \\
& =\mathbb{P}\{x-\xi \leq \sqrt{t}, \xi \leq x\}+\mathbb{P}\{\xi-x \leq \sqrt{t}, \xi>x\} \\
& =\mathbb{P}\{x-\sqrt{t} \leq \xi \leq x\}+\mathbb{P}\{x<\xi \leq x+\sqrt{t}\}
\end{aligned}
$$

with

$$
\begin{aligned}
& \mathbb{P}\{x-\sqrt{t} \leq \xi \leq x\}= \begin{cases}\sqrt{t} /(2 \delta) & \text { if } \sqrt{t}<x+\delta \\
(x+\delta) /(2 \delta) & \text { if } \sqrt{t} \geq x+\delta,\end{cases} \\
& \mathbb{P}\{x<\xi \leq x+\sqrt{t}\}= \begin{cases}\sqrt{t} /(2 \delta) & \text { if } \sqrt{t}<\delta-x \\
(\delta-x) /(2 \delta) & \text { if } \sqrt{t} \geq \delta-x .\end{cases}
\end{aligned}
$$

This yields the expression (42) for $F_{x, \delta}(t)$ in the case $|x| \leq \delta$.

If $x>\delta$ then $\eta_{x, \delta} \geq(x-\delta)^{2}$ with probability 1 implying $F_{x, \delta}(t)=0$ for all $t \leq(x-\delta)^{2}$ and $\mathbb{P}\{x<\xi \leq x+\sqrt{t}\}=0$ for all $t$. Therefore

$$
F_{x, \delta}(t)=\mathbb{P}\{x-\sqrt{t} \leq \xi \leq x\}= \begin{cases}0 & \text { if } \sqrt{t} \leq x-\delta \\ \frac{\delta-(x-\sqrt{t})}{2 \delta} & \text { if } x-\delta<\sqrt{t}<x+\delta \\ 1 & \text { if } \sqrt{t} \geq x+\delta,\end{cases}
$$

This yields the expression (42) for $F_{x, \delta}(t)$ in the case $|x|>\delta$.

Deduction of the formulas (43) for the density and (44) for the moments from the expression (42) for the c.d.f. $F_{x, \delta}(t)$ is an easy exercise. 
Lemma 2 Let $\delta>0, x \in \mathbb{R}$ and $\eta_{x, \delta}^{\prime}$ be a r.v. $\eta_{x, \delta}^{\prime}=|\xi-x|$, where r.v. $\xi$ has uniform distribution on $[-\delta, \delta]$. Then the c.d.f. of the r.v. $\eta_{x, \delta}^{\prime}$ is

$$
F_{x, \delta}^{\prime}(t)=\mathbb{P}\left\{\eta_{x, \delta}^{\prime} \leq t\right\}= \begin{cases}0 & \text { for } t \leq 0 \\ \frac{t}{\delta} \cdot 1_{[|x| \leq \delta]} & \text { for } 0<t<|\delta-| x|| \\ \frac{\delta-|x|+t}{2 \delta} & \text { for }|\delta-| x|| \leq t \leq \delta+|x| \\ 1 & \delta+|x|<t,\end{cases}
$$

The corresponding density of $\eta_{x, \delta}^{\prime}$ is

$$
\varphi_{x, \delta}^{\prime}(t)= \begin{cases}\frac{1}{\delta} \cdot 1_{[|x| \leq \delta]} & \text { for } 0<t<|\delta-| x|| \\ \frac{1}{2 \delta} & \text { for }|\delta-| x||<t \leq \delta+|x| \\ 0 & \text { otherwise. }\end{cases}
$$

Lemma 2 follows from Lemma 1 by noting that $\eta_{x, \delta}^{\prime}=\sqrt{\eta_{x, \delta}}$.

Note that $1_{[|x| \leq \delta]}=0$ for $|x|>\delta$ and one of the two non-trivial cases in (42), (43), (47) and (48), when $|x|>\delta$, become trivial as expressions vanish to zero.

\section{References}

1. Blum A, Hopcroft J, Kannan R (2020) Foundations of data science. Cambridge University Press, Cambridge

2. Janson S (1986) Random coverings in several dimensions. Acta Mathematica 156:83-118

3. Januszewski J, Lassak M (1994) On-line covering the unit cube by cubes. Discrete Comput Geom 12(4):433-438

4. Joe S, Kuo FY (2008) Constructing Sobol sequences with better two-dimensional projections. SIAM J Sci Comput 30(5):2635-2654

5. Johnson ME, Moore LM, Ylvisaker D (1990) Minimax and maximin distance designs. J Stat Plan Infer 26(2):131-148

6. Kuperberg W (1994) On-line covering a cube by a sequence of cubes. Discrete Comput Geom 12(1):83-90

7. Li S (2011) Concise formulas for the area and volume of a hyperspherical cap. Asian J Math Stat 4(1):66-70

8. Niederreiter H (1992) Random number generation and quasi-Monte Carlo methods. SIAM, Philadelphia

9. Noonan J, Zhigljavsky A (2021) Non-lattice covering and quantization in high dimensions. In: Black Box Optimization, Machine Learning and No-Free Lunch Theorems. Springer

10. Petrov VV (1975) Sums of independent random variables. Springer, Berlin

11. Petrov VV (1995) Limit theorems of probability theory: sequences of independent random variables. Oxford Science, Oxford

12. Prakasa Rao BLS (1987) Asymptotic theory of statistical inference. Wiley, New York

13. Pronzato L, Müller W. G. (2012) Design of computer experiments: space filling and beyond. Stat Comput 22(3):681-701

14. Sukharev A (1992) Minimax models in the theory of numerical methods. Springer Science \& Business Media, Berlin

15. Tibken B, Constales D, et al. (1997) The volume of the intersection of a concentric cube and ball in n-dimensional space: collection of approximations. In: SIAM Review, vol 39, pp 783-786

16. Tóth GF (2017) Packing and covering Handbook of discrete and computational geometry. Chapman and Hall, London, pp 27-66 
17. Tóth GF, Kuperberg W (1993) Packing and covering with convex sets. In: Handbook of Convex Geometry. Elsevier, pp 799-860

18. Zhigljavsky A (1991) Theory of global random search. Kluwer, Norwell

19. Zhigljavsky A, Zilinskas A (2007) Stochastic global optimization. Springer Science \& Business Media, Berlin

20. Žilinskas A (2013) On the worst-case optimal multi-objective global optimization. Optim Lett 7(8):1921-1928

Publisher's Note Springer Nature remains neutral with regard to jurisdictional claims in published maps and institutional affiliations. 